UNIVERSTTE DO QUEBEC

\author{
MEMOIRE \\ PRESENTE A \\ L'UNIVERSITE DU QUEBEC A CHIOOUTIMI \\ OOMME EXIGENCE PARTIELIE \\ DE IA MAITTISE EN GESTION DES P.M.O.
}

PAR

IAVAL OUEILET

PIANIFICATION D' ON LOTISSEMENT DOMICIIIAIRE

JUIN 1990 


\section{Bibliothèque}

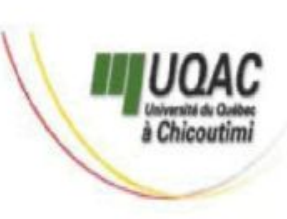

\section{Mise en garde/Advice}

Afin de rendre accessible au plus Motivated by a desire to make the grand nombre le résultat des results of its graduate students' travaux de recherche menés par ses research accessible to all, and in étudiants gradués et dans l'esprit des accordance with the rules règles qui régissent le dépôt et la governing the acceptation and diffusion des mémoires et thèses diffusion of dissertations and produits dans cette Institution, theses in this Institution, the I'Université du Québec à Université du Québec à Chicoutimi (UQAC) est fière de Chicoutimi (UQAC) is proud to rendre accessible une version make a complete version of this complète et gratuite de cette œuvre. work available at no cost to the reader.

L'auteur conserve néanmoins la The author retains ownership of the propriété du droit d'auteur qui copyright of this dissertation or protège ce mémoire ou cette thèse. thesis. Neither the dissertation or Ni le mémoire ou la thèse ni des thesis, nor substantial extracts from extraits substantiels de ceux-ci ne it, may be printed or otherwise peuvent être imprimés ou autrement reproduced without the author's reproduits sans son autorisation. permission. 
'AVANT-PROPOS ET REMERCIEMENTS

Ce projet, qui est devenu réalité est dû aux conseils, au dévouement et à la gentillesse de certaines personnes.

La première personne qui $\mathrm{m}^{\prime a}$ secondé, encouragé, est mon associé, monsieur Gaston-L. Tremblay. Je ne pourrai jamais le remercier assez des connaissances, de son expérience qu'il a cherché à me transmettre. Il m'a témoigné une grande confiance et de l'amitié.

Je tiens à remercier, monsieur Gilles G. Bergeron, superviseur du mémoire qui m'a indiqué clairement, sans ambages, les étapes à franchir pour réaliser cette recherche-action. Ce mémoire a contribué à la réussite du projet.

En terminant, qu'il me soit aussi permis d'exprimer ma gratitude envers les membres du Comité de lecture, messieurs Claude Lalonde et Robert Nelson. 
Liste des documents consultés..................... III

Liste des annexes........................... VI

Introduction. ............................ 1

Chapitre I Définition et faisabilité du projet............ 3

1.1 Objet de $l^{\prime}$ étude.......................... 3

1.2 Faisabilité: Examen préliminaire............... 5

1.3 Rentabilité......................... 7

1.4 Forces et faiblesses du projet................. 9

Chapitre 2 Étude du marché.................... 12

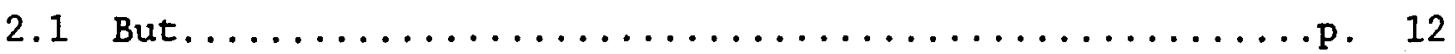

2.2 Analyse situationnelle du marché................ 12

2.3 Données sur les permis de construction de 80 à $87 \ldots \ldots$. p. 14

2.4 Profil socio-économique des nouveaux résidents par quartier......................... 15

Chapitre $3 \quad$ Choix du site...................... 22

3.1 Situation géographique..................... 22

3.2 Aspect visuel......................... 25

3.3 L'environnement......................... 25

3.4 Superficie du terrain..................... 26

3.5 Les qualités du terrain..................... 26

3.6 Services d'égout, aqueduc et électricité........... 28

3.7 Coût du terrain....................... 28

Chapitre 4 Analyse de la problématique............... 30

$4.1 \quad$ L'organisation..................... 30

4.1.1 Structure organisationnelle.............. 33

4.1.2 Structure financière................. 33

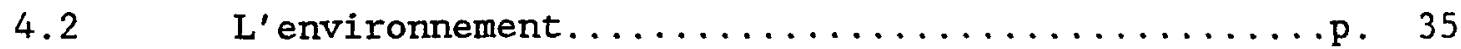

4.2.1 L'environnement physique.............. 35

4.2.2 L'environnement économique.............. 36

4.2.3 L'environnement politique.............. 38 
4.3 Description du processus................. 41

$4.3 .1 \quad L^{\prime}$ achat du terrain.................... 41

$4.3 .2 \quad$ La géologie....................... 43

4.3 .3 Le plan d'aménagement.................. 45

4.3 .4 Le plan de financement.................. 47

Chapitre $5 \quad$ Les problèmes secondaires................ 49

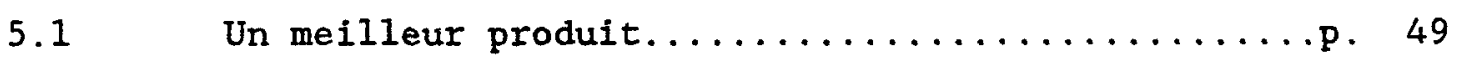

5.1 .1 Le dessin des rues.................... 50

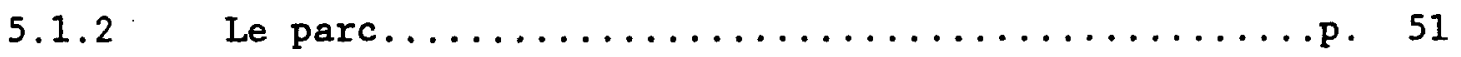

5.1 .3 La réglementation.................... 51

$5.1 .4 \quad$ L'enfouissement des fils électriques.......... 53

5.1 .5 Le mobilier urbain.................... 54

5.2 Le marketing.................... 55

5.2 .1 Le produit..................... 55

5.2 .1 .1 Le positionnement du produit............... 56

$5.2 .2 \quad$ La publicité................... 57

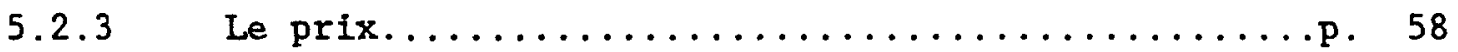

$5.2 .4 \quad$ La force de vente.................... 59

Chapitre $6 \quad$ Modèle conceptuel.................... 61

$6.1 \quad$ Le modèle....................... 62

6.2 Application du modèle................. 63

$6.2 .1 \quad$ Terrain........................ 63

$6.2 .2 \quad$ Achat...................... 64

$6.2 .3 \quad$ Faisabilité........................ 64

$6.2 .4 \quad$ Plan de lotissement................... 65

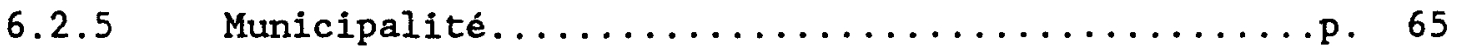

$6.2 .6 \quad$ Construction........................ 66

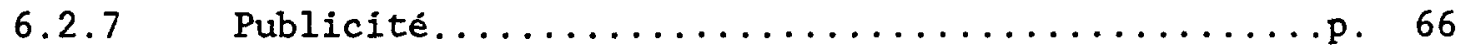

$6.2 .8 \quad$ Vente........................ 67

$6.2 .9 \quad$ Financement..................... 67

Conclusion. ............................... 69 


\section{LISTE DES DOCUMENTS CONSULTES}

Brackan, Ian. Urban planning methods. London, Methen, 1981, 400 pages.

Darmon, R.Y.; Laroche, M.; Petraf, J.V. Le Marketing fondements et applications. Montréal, Mc Graw Hill, 1978, 442 pages.

Divay, Gérard. La formation des espaces résidentiels, Montréal, Presses de 1 'Université du québec, 1984, 262 pages.

Jeanneret, Edouard (Le Corbusier). L'urbanisme. Paris Arthaud 1980, 284 pages.

Lavoie, Claude. Initiation a l'urbanisme. Montréal, Editions Georges Le Pape, 1978, 150 pages.

S.C.H.L. Le choix d'un modele de maison. Publications officielles, Ottawa, 1973, 48 pages.

Winter, Eric. Urban landscapes, Scarborough, Bellhaven House, 1969, 148 pages.

Wogensky, André. Architecture active. Tournai, Casterman 1972, 258 pages. 


\section{ARTICLES DE JOURKAUX}

Dubois, Robert. "Le quartier avant la maison; n'allez pas vivre n'importe où".

La Presse 02-01-88 cahier $J$

Dubois, Robert. "Le choix du terrain".

La Presse 05-03-88 cahier $C$

Dubois, Robert. "L'emplacement est la clé du succès immobilier". La Presse 10-12-88 cahier J

Dubois, Robert. "Où acheter une propriété".

La Presse 25-03-89 cahier J2

Lévesque, Lia. "Chicoutimi est la région la plus francophone du Québec: données de Statistiques Canada concernant le bilinguisme, l'immigration et la valeur des maisons au Québec".

La Presse 30-01-89 cahier C9 
LISTE DES ANNEXES

I Sondage sur les tendances en habitation à Chicoutimi.

II Profil du résident: Quartier Alfred Boivin.

III "

IV "

$\mathrm{V} \quad *$

VI "
": Domaine du Roy.

" : Quartier Modèle.

": Rue des Maristes.

": Quartier Gobeil.

VII Règlements de construction pour "La Seigneurie des Lilas".

VIII Rapport de construction, septembre 1988, Service d'urbanisme, Ville de Chicoutimi. 


\section{INTRODUCTION}

L'espace résidentiel est un facteur agissant sur notre personne. Notre habitation et son environnement influencent notre qualité de vie.

De plus en plus de gens accordent de l'importance à ce sujet. On recherche une habitation confortable, décorée, dans un environnement agréable. Les tendances actuelles visent à satisfaire ce besoin, étant donné que le niveau économique de la population s'accrôt et que 1'investissement, dans ce besoin primaire, se révèle généralement intéressant tout en permettant de jouir du bien dans le présent.

Le désir d'améliorer sa demeure stimule la construction, la rénovation et l'achat de propriété existante. Nous nous intéresserons à la construction d'habitation dans cet ouvrage, plus spécifiquement au lotissement d'espace requis pour la construction.

Nous chercherons dans la première partie à connaître les besoins des individus afin de produire un lotissement répondant à leurs attentes. Par la suite, nous effectuerons la démarche de conception et d'implantation du quartier. 
Cette étude est une véritable recherche-action. Le projet existe, certains problèmes demanderont beaucoup de temps à être résolus comparativement à d'autres, ceci sans égard à l'importance du problème. Il faudra composer avec la réalité.

Les plus grandes difficultés à résoudre sont les situations où la décision est extérieure à l'entreprise, comme les fonctionnaires municipaux, les politiciens...

Ces négociations constituent des embûches où le projet est souvent remis en question. La planification doit absolument tenir compte de cette réalité pour réussir. Pour le lecteur, certains problèmes soulevés paraitront peu importants, mais parfois ils ont constitué le "talon d'Achille" du projet.

L'auteur a voulu exposer la démarche poursuivie du début du projet jusqu'au début de la réalisation des travaux de lotissement. C'est la véritable phase de promotion: être l'instigateur et assurer son cheminement. Cette étape demande persévérance, ténacité, réflexion, bon sens. Espérons que le texte qui suit révélera au lecteur ces deux derniers points: réflexion et bon sens. 
CHAPITRE I

DÉFINITION ET FAISABILTTÉ DU PROJET.

\subsection{OBJET DE L'ÉTÜDE}

Le but de ce mémoire est d'élaborer une démarche visant à développer un environnement résidentiel de qualité. Ce quartier projeté comprendra une centaine de résidences familiales.

Nous tenons à spécifier que la qualité de réalisation du lotissement est un aspect très important de ce projet. L'entreprise souhaite que ce quartier démontre un environnement agréable, respectant les règles de l'urbanisme d'aujourd'hui.

Pour ce faire, le plan de lotissement doit exploiter avantageusement la géographie du terrain et déterminer une réglementation concernant la construction pour une harmonisation des bâtiments. Cette étape du projet devra s'inspirer des réalisations les plus valables tout en l'adaptant à notre environnement.

Avant le début de la première maison, une planification de l'ensemble du lotissement doit être élaborée afin de maximiser les 
chances de réussite du but projeté. Le lotisseur devra s'occuper de différents points: le dessin des rues, l'aménagement des parcs, le mobilier urbain et l'harmonisation des résidences.

Ce dernier point est très important et n'est pas directement déterminé par le lotisseur, car celui-ci ne veut pas construire les résidences. Son désir est de vendre le terrain au résident et ce dernier aura la responsabilité de construire.

Les promoteurs devront rédiger un cahier de réglementation que le constructeur devra respecter pour développer une harmonie visuelle.

La qualité, la valeur de chaque résidence est reliée à l'ensemble des habitations. Le propriétaire d'une résidence a tout à gagner à se conformer au plan d'ensemble. Chaque propriétaire sera gagnant. L'appréciation de la valeur de sa propriété dépend de son environnement dans une certaine partie. Ce contrôle de l'aspect extérieur des constructions déterminera un environnement planifié, respectant les règles de l'esthétisme.

Ces règles devront être réalistes, applicables et acceptées de bơn gré des propriétaires. La vision des promoteurs doit être exprimée clairement, simplement, pour être comprise et véhiculée par la suite par les propriétaires. 
Les objectifs des promoteurs doivent respecter ceux des propriétaires. Une étude de marché déterminera les désirs, les attentes des clients potentiels. Une clientèle-cible sera déterminée en tenant compte des objectifs fixés.

Le promoteur doit être capable de définir le plan d'ensemble, d'expliquer ses contraintes et de justifier les observations qui appuient ce plan.

La relation entre qualité de l'environnement et qualité de vie est une prémisse admise de chacun. Les efforts pour construire un bel environnement donneront un résultat permanent. Une planification réfléchie, étudiée, permettra de construire un tel lieu de vie urbain agréable. Le règlement d'architecture des résidences devra permettre à chacun de satisfaire ses besoins, ses goûts, tout en respectant les critères de l'harmonie visuelle.

\subsection{FAISABILITE: EXAMIEN PRÉLIMINAIRE}

Une partie du travail du promoteur est d'aiguiller l'action pour satisfaire chaque intervenant. Une confiance mutuelle est requise dans la majorité du temps. Les participants au projet doivent croire à sa réussite. 
Dans ce projet, les promoteurs veulent jouer un rôle de coordonnateur.

L'étude du sol, le lotissement, le cadastrage sont les premiers déboursés à effectuer pour commencer le projet.

Lorsque ces étapes seront complétées, la construction des rues et l'acquisition du terrain devront faire l'objet d'une entente satisfaisant le constructeur, les vendeurs et les promoteurs. Deux options s'offrent à ces derniers. La première est la collaboration du constructeur et des vendeurs. Le paiement est fait au fur et à mesure que les entrées de fonds découlant de la vente des terrains s'effectuent.

La seconde est de s'associer à un partenaire financier de type institutionnel. Cette solution diminuera le profit, mais l'entreprise demeure entièrement sous le contrôle des actionnaires.

On remarque à ce stade-ci que l'habileté des promoteurs à négocier de bonnes ententes avec ces intervenants dans le projet est primordiale à la réussite du projet.

C'est la première solution appelée communément "tour de table" qui a été privilégiée par les promoteurs. Les mises de fonds et parfois le risque sont supportés par les autres. La confiance dans les promoteurs est essentielle pour arriver à ériger une telle 
opération. Dans notre cas, développer une parcelle du projet la première année va diminuer la charge monétaire de construction. Il est non rentable de penser à tout lotir en une seule opération.

Selon le plan de lotissement, un premier secteur englobant une vingtaine de terrains sera développé pour la première année, et dans les deux autres années qui suivront, le développement se fera par phases. L'étude du potentiel de vente nous éclairera davantage sur cette estimation.

\subsection{RENTABILITE}

Pour nous permettre d'avoir une approximation du bénéfice de l'opération, nous allons décomposer théoriquement le prix d'un terrain de 7,000 pieds carrés vendu 20000 dollars.

Certaines données de cette prévision sont précises tandis que d'autres sont basées sur des barèmes théoriques. 


\subsubsection{Dépenses foncières}

- terrain

$7000 \$$

- charges de lotissement

charges cadastrales

$300 \$$

- taxe de mutations

- taxes municipale et scolaire sur une

période de six mois

\subsubsection{Dépenses de constructions}

- travaux payés à l'entrepreneur

$8000 \$$

\subsubsection{Dépenses annexés}

- frais de gestion ( $7 \%$ X $8000 \$$ )

$560 \$$

- frais de vente ( $5 \%$ X 18 000\$)

$900 \$$

\subsubsection{Prix de revient}

- somme de $1+2+3$

$17003 \$$

\subsubsection{Bénéfice}

Prix de vente

$20000 \$$

Prix de revient

$17003 \$$

Bénéfice

$2997 \$$

Ce bénéfice représente $2997 / 17003$, soit 17.68 du prix de revient.

Cette étude "pro-forma" donne une idée du bénéfice auquel on peut s'attendre. Il va de soi que si le prix de vente est plus élevé, le pourcentage sera beaucoup plus important. Les dépenses de constructions peuvent aussi être plus importantes également.

Pour avoir discuté de ce sujet avec d'autres bâtisseurs, un profit de 15 à $20 z$ s'avère être la norme habituelle. 


\subsection{FORCES ET FAIBLESSES DU PROJET}

\section{Marketing}

Avantages géographiques du terrain:

- proximité des institutions, fermé, site nature1;

- qualité du produit: architecture urbaine appropriée;

- planification du marketing.

Désavantages:

- concurrence forte;

- rumeur négative sur la qualité du sol;

- vacance du loyer;

- construction de HLM.

\section{Production}

La qualité du développement peut être supérieure à ce qui a été fait jusque-1à, car on peut s'inspirer des développements passés et surtout des meilleures réalisations dans d'autres villes. 


\section{Personne1}

Les deux associés forment une équipe qui se complète mutuellement. Un associé a beaucoup d'expertise en développement, et est avantageusement connu dans le milieu; l'autre, peu connu, est disponible pour investir beaucoup d'heures de travail.

Les deux poursuivent le même but, soit une réalisation de qualité.

\section{Finance}

La démarche de réalisation du projet devra se poursuivre de manière qu'aucune contrainte financière ne nuise à sa réalisation.

Pour ce faire, les promoteurs misent sur une planification des déboursés échelonnée selon les phases de réalisation.

Les points à exploiter: Les avantages dans le marché.

1. La proximité du quartier; on retrouve, dans un périmètre possible à la marche: une école élémentaire, secondaire pour garçons, collégiale, universitaire, un hôpital, deux centres commerciaux et bien d'autres commodités. 
2. Un espace de développement borné d'arbres, de talus. L'espace est ouvert. Le site gardera cet aspect naturel après l'aménagement.

3. L'entrée dans ce quartier doit obligatoirement passer dans un espace défini: il peut $y$ avoir au maximum deux rues qui conduisent à l'intérieur du quartier. Ceci peut créer un aspect différent des autres quartiers de la ville.

4. Le désir de réaliser un beau développement est une autre force. Les conditions physiques le permettent également. Le profit n'est pas l'unique aspect qui conditionne ce développement.

5. En s'inspirant du passé, des développements qui se font ailleurs, il serait normal de réussir un développement plus moderne, satisfaisant les besoins de ses résidents. 


\section{CHAPITRE 2}

\section{2. ÉTUDE DU MARCHÉ}

\subsection{But}

Par cette étude, nous nous proposons d'analyser le marché de la construction résidentielle unifamiliale dans certains secteurs de Chicoutimi et de déterminer le profil du consommateur.

\subsection{Analyse situationnelle du marché}

A chaque année, nous voyons apparaître de nouvelles maisons dans différents quartiers. Les questions pertinentes à nous aider dans notre démarche de lotissement peuvent trouver réponse dans l'analyse de la clientèle d'acheteurs de ces maisons.

\footnotetext{
Pour bénéficier d'une analyse de marché judicieuse, il est important d'avoir des questions claires, précises et concises qui vont permettre de déterminer la taille, la structure du marché et le profil des gens qui le composent.

Notre analyse se base sur le passé. Etant donné que notre projet est à moyen terme, (deux à trois ans), nos extrapolations seront basées sur les données précueillies
} 
dans la dernière décennie sans tenir compte des variables futures. Nous chercherons à cerner la réalité présente.

Selon 1'inspecteur chef en bátiment de la ville de Chicoutimi, 50 à 608 des permis sont demandés dans deux secteurs particuliers de la municipalité: Rivière-duMoulin, et le secteur sud comprenant les nouveaux lotissements près des centres commerciaux et vers le boulevard St-Paul. Il se bâtirait une centaine de maisons unifamiliales par année dans ce territoire. Si nous prenons en considération les résultats des années passées, nous pouvons projeter qu'au cours des deux ou trois prochaines années ce nombre devrait se maintenir si la situation économique demeure semblable.

Une augmentation substantielle du taux d'intérêt pourrait perturber la projection, mais les prévisions économiques ne nous permettent pas d'extrapoler dans un sens ou dans l'autre. L'économie étant variable, nous devrons toujours prendre des décisions sécures, n'engager des sommes que par étapes, soit lotir une partie et la vendre avant d'entreprendre un autre chantier.

Un tel mode de décision permettra de s'ajuster au marché et diminuera les risques d'investissement. 


\subsection{Données sur les permis de construction de 80 a 87}

Nombre de permis

Unifamiliale

$1980\lceil 1981\lceil 1982\lceil 1983\lceil 1984\lceil 1985\lceil 1986\lceil 1987$

- - - - - +- -

Bifamiliale

| $174|126| 74|207| 192|157| 181|191|$

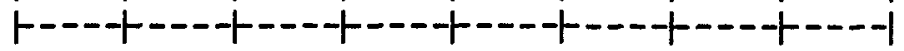

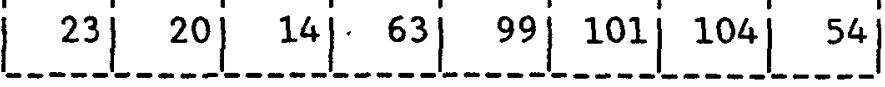

Valeur déclarée (en milliers de dollars)

\begin{tabular}{|c|c|c|c|c|c|c|c|c|}
\hline & 1980 & 1981 & 1982 & 1983 & 1984 & 1985 & 1986 & 1987 \\
\hline hifamiliale & & 5941 & 3526 & 10006 & 10957 & 10386 & 12318 & 13338 \\
\hline ifamiliale & 1064 & 1066 & 731 & 3444 & $\begin{array}{lll}6 & 035\end{array}$ & 6491 & 7190 & 3927 \\
\hline
\end{tabular}

Réf.: Rapport de constructions: Service d'urbanisme, Ville de Chicoutimi.

La taillé du marché ne varie pas beaucoup dans l'unifamiliale, exception faite de la période de récession économique 1981 et 1982 . Ces résultats couvrent l'ensemble de la municipalité: secteur Nord, Rivière-du-Moulin, paroisse et secteur sud.

Notre projet se situant dans le secteur sud, nous analyserons plus spécialement ce secteur ainsi que celui de Rivière-du-Moulin, situé à proximité. 


\subsection{Profil socio-économique des nourvaux résidents par quartier}

Etant donné la facilité de répertorier les nouveaux résidents, un sondage écrit a été effectué par quartier. Le but était de tracer le profil moyen du client et de connaitre son point de vue sur l'achat d'un terrain, le choix du quartier.

Nous avons délimité la clientèle visée par ce questionnaire aux nouveaux espaces résidentiels. Vous trouverez en annexe le questionnaire et les résultats pour chaque nouveau lotissement étudié. Cette enquête a été effectuée en automne 1987.

\subsubsection{Age}

Ces personnes sont âgées de vingt-cinq à quarante ans. Nous retrouvons bien sûr quelques acheteurs plus ágés constituant une autre classe mais moins importante que les 25-40.

\subsubsection{Situation familiale}

Ces personnes vivent en famille dont la plupart ont un ou deux enfants, 20z n'ont pas d'enfants et un autre 208 ont trois ou quatre enfants.

\subsubsection{Revenu}

\subsubsection{Revenu principal}

La moyenne du revenu principal annuel est de 35 000\$. $30 \%$ ont déclaré un revenu de $45000 \$$ et plus. 


\subsubsection{Revenu familial}

La majorité de ces ménages (65\%) ont deux revenus. La moyenne obtenue inclut les ménages à un seul revenu également: elle se situe entre 40000 et 50000 s dollars. Si on ne tient compte que des couples ayant chacun un emploi, le revenu moyen par famille grimpe entre 50 000\$ et 60 000s.

\subsubsection{Type d'emploi}

Le quart de ces personnes travaillent à leur compte, la moitié pour l'entreprise privee et la derniere partie pour 1 'Etat.

\subsubsection{Prix pour un terrain}

Le prix moyen se situait à 20663 . On retrouvait $20 \%$ de la clientèle étant prête à payer 25000 s et un autre 20\% déclarant être prêt à débourser 30 000\$ et plus.

\subsubsection{Superficie du terrain}

La moitié du groupe désire des terrains de 7000 pieds carrés et moins. Quelque 37\% désirent entre sept et 10000 pieds carrés. 
2.4.7 Prix pour une résidence

La moyenne obtenue du prix est de 100 000\$. La majorité (638) ne veut pas payer plus que ce montant. Quelques $30 \%$ sont d'accord entre $100000 \$$ et $150000 \$$.

\subsubsection{Propriétés antécédentes}

Pour 228 de ce groupe, c'est leur première maison, 40\% leur deuxième maison, 168 leur troisième. Pour trois personnes, c'est leur sixième, septième et huitième maison.

\subsubsection{Choix du quartier}

Nous avons essayé de cerner les points qui influencent dans la décision du choix du quartier. L'aspect général du quartier est un critère important dans la décision. La qualité du voisinage est analysée également par 578 des gens.

Le prix du terrain et des maisons a une importance relative dans le choix, tout comme ia proximité du lieu de travail.

Etre proche des écoles, services communautaires, centres commerciaux, n'est guère pris en considération par les gens.

Ce dernier point est étonnant, et mérite quelques explications.

Dans les traités d'urbanisme, on reconnait que la proximité des facilité communautaires, 
culturelles et récréatives est un point important, avantageux et recherché des habitants d'une ville.

Comment expliquer que les résultats de l'enquête démontrent des résultats contraires à la théorie? Nous tenterons deux justifications:

Chicoutimi est une ville où se déplacer d'un point à l'autre ne crée aucun problème aux résidents. Seulement $25 \%$ des propriétaires de nouvelles maisons sont influencés par ce point. De plus, de nombreuses familles ont deux automobiles dans notre région.

Une autre explication suggérée est que, tout simplement, les réponses obtenues lors de l'enquête reflètent la situation du répondant: c'esta-dire que si la maison est loin des facilités, il répondra que c'est peu important pour lui.

Nous croyons par contre que ce point est un critère important dans le choix du quartier. La valeur d'une maison n'est la même d'un quartier à l'autre. Certains quartiers de Chicoutimi sont plus recherchés et les maisons se vendent à prix plus élevé.

Un quartier bien situé demeure un avantage apprécié et contribue à la réussite d'un projet.

\subsubsection{Points incitant a I'augmentation des prix $^{\prime}$}

Les gens seraient disposés à payer plus cher un terrain si on augmente sa superficie. Un 
terrain boisé est un autre atout pour une majoration des prix; tandis que la proximité des services ne semble pas constituer une raison de payer davantage pour un terrain. Seulement 158 des répondants seraient prêts à débourser davantage pour habiter près des services.

2.4.11 Position quant a une réglementation sur la construction des maisons

La très grande majorité (90\%) est en faveur d'une réglementation sur la construction. $\mathrm{Ce}$ point est très marqué. Nous pouvons penser qu'une réglementation assujettie dans un lotissement peut être un incitatif lors de la vente de lot.

\subsubsection{Réglementation conforme}

La question manquait d'explications. La moitié des gens ont répondu en faveur d'une réglementation précise, I'autre moitié en désaccord.

\subsubsection{Points à réglementer}

La majorité des personnes ( 50 à 60\%) est d'accord pour réglementer la période de temps pour aménager le terrain (gazon, entrée, etc.) et l'architecture des maisons. Seulement le quart du groupe est d'avis que les règlements doivent porter sur les couleurs et les matériaux de recouvrement. 
Nous venons de tracer le profil basé sur la moyenne de la clientèle sondée dans l'ensemble des nouveaux lotissements que nous avons désignés auparavant. Vous retrouverez en annexe le profil basé sur la moyenne des réponses obtenues par nouveau quartier.

Pour appuyer également la projection sur les nouvelles constructions, nous joignons en annexe le rapport de construction de septembre 88, ainsi que les autres rapports qui ont servi à établir cette projection.

En résumé, à la suite de ce sondage, nous croyons qu'à Chicoutimi, environ 30 clients sur une possibilité de 125 à 150 seraient prêts à débourser $25000 \$$ et plus pour 1'achat d'un terrain de superficie d'au moins 7000 pieds carrés.

Ces gens âgés de vingt-cinq à quarante ans ont déjà une propriété. Ils recherchent un environnement de qualité. Ils sont favorables à une réglementation sur la construction pour qu'une harmonie visuelle se dégage du nouveau lotissement, réglementation ne devant pas être excessive.

L'objectif des promoteurs est de réaliser un beau quartier, et ils désirent le plus possible accueillir les clients disposés à investir une somme d'argent plus élevée que la moyenne des résidents de la municipalité.

La cible est la partie supérieure du marché, ceux qui sont prêts à débourser 25000 st plus. L'importance de ce marché est suffisante pour le projet si l'échéancier est de trois ans. Ces clients disposés à débourser un prix plus élevé construiront une maison plus importante que la moyenne. 
Le sondage a été réalisé dans des quartiers de "moyenne" et "haut de gamme", où l'on retrouve des maisons qui conviendraient aux attentes. Il n'a pas été tenu compte des résidences "bas de gamme".

La prévision est de vendre une vingtaine de terrains pour construction unifamiliale par année à prix plus élevé que la moyenne, soit de $20000 \$$ et plus. Cette prévision permet de croire qu'en lotissant vingt terrains, ils seront vendus dans un délai d'un an. Et si cette prévision se révèle inexacte, il sera possible de reculer ou d'avancer le début des travaux de la seconde phase. L'entreprise sera toujours dans des conditions financières sécuritaires. II va sans dire que plus les ventes se font rapidement, mieux c'est, mais on doit tenir compte de la taille du marché dans un tel projet. 


\section{CHAPITRE 3}

\section{CHOIX DU STTE}

Le secteur choisi pour ériger un quartier a une très grande importance dans la réussite d'un projet. On doit chercher à répondre le mieux possible aux exigences des futurs résidents.

Comme nous $l^{\prime}$ avons décrit précédemment, notre intention est de faire un quartier de "qualité". L'emplacement devra répondre à des critères précis pour maximiser les chances de réussite.

\subsection{Situation géographique}

La croissance d'une ville se fait le plus souvent en élargissant sa périphérie. Le terrain près de son centre est occupé et contient généralement les services à la communauté.

La ville de Chicoutimi a présentement deux noyaux où se centralisent ses services communautaires: la rue Racine, et le boulevard Talbot à la hauteur des centres commerciaux.

Dans cette zone géographique, nous retrouvons des écoles de tout niveau, des garderies, les installations communautaires (hôpital, églises, centres commerciaux), culturelles (bibliothèque, salle de spectacle, cinéma, musée) et récréationnelles (parcs, terrains de jeux, piscines, centres sportifs). 
Habiter près de ces services constitue pour beaucoup de personnes un avantage apprécié. Généralement, les personnes préfèrent se déplacer rapidement d'un point à l'autre et avoir un grand choix dans les services recherchés.

Le terrain retenu pour ce projet répond bien à ces critères. Dans la figure qui suit, nous avons déterminé les distances pour chacun des services énumérés précédemment. 
Figure 3.1

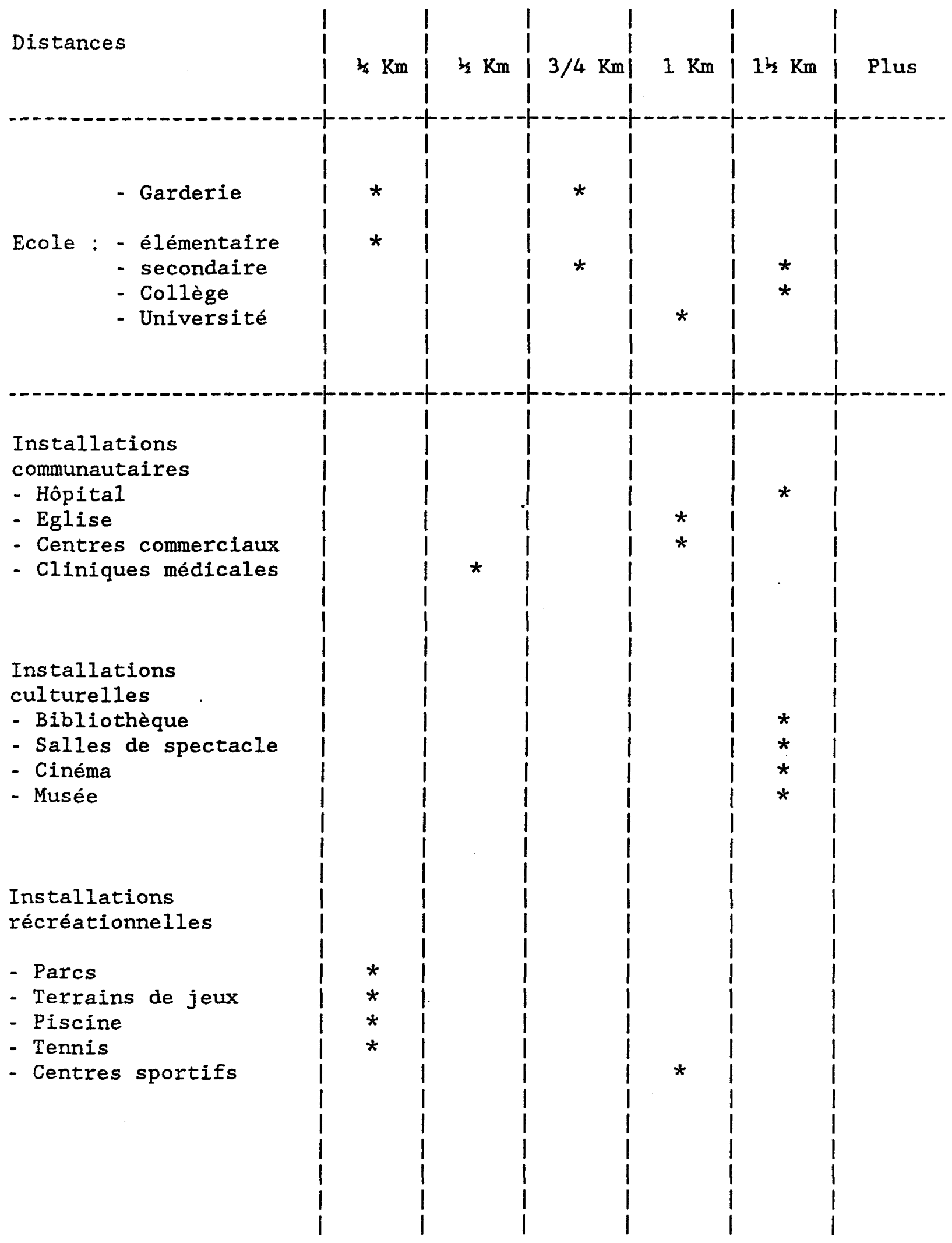




\subsection{Aspect visuel}

Le décor naturel d'un site est un autre point déterminant. On peut agir sur l'aménagement du terrain. Au point de départ, le terrain doit offrir une certaine qualité visuelle.

Le terrain projeté est délimité par un boisé. C'est une bande de terre dans une vallée prononcée. Cette bande est légèrement plus basse que le sommet.

Cette situation par contre, protège contre les vents et permet un bon drainage. Le relief accidenté produit un décor boisé. L'étroitesse de la bande fait que la plupart des terrains résidentiels $n^{\prime}$ auront pas de voisin arrière, qualité recherchée par plusieurs.

L'aménagement du terrain devra démontrer une conceptualisation soignée. Le dessin des rues la reflétera. L'aspect visuel des maisons devra également être réglementé pour chercher 1'harmonie visuelle.

\subsection{L'environnement}

L'entourage du quartier permet de s'approprier la réputation de son voisinage. Certains secteurs sont plus réputés que d'autres. Nous retrouvons à Chicoutimi quelques quartiers ayant une certaine réputation: rue du Séminaire, Notre-Dame-du-Saguenay, Domaine du Roy, Panoramique.

Développer un secteur près de ces quartiers permet de s'approprier un peu de leur "prestige". 
Dans notre cas, le terrain est dans le quartier Murdock, tout près de Notre-Dame-du-Saguenay: nous considérons ce point avantageux pour le lotissement. Le voisinage est d'une certaine qualité.

Un point négatif est la présence d'un boulevard qui provoque du bruit. L'absence de bâtisse fait qu'au début cet inconvénient est plus marqué. Ce point ne met pas en péril le projet, car il existe des solutions pour régler ce problème, comme des barrières acoustiques faites de béton ou une plantation de conifères sur un talus.

\subsection{Superficie du terrain}

Ce terrain de $900000 \mathrm{pi}^{2}$ permet d'accorder une certaine ampleur au projet. On croit qu'une centaine d'habitations pourront y être érigées. Ce nombre permet d'effectuer des études car ces dépenses réparties sur chaque terrain n'affecteront pas trop la rentabilité du projet, qu'on pense aux frais d'urbanistes, de géologues.

La superficie exige également un échéancier de développement. L'étude de marché déterminera la vitesse de développement. Chaque année, une partie seulement de la superficie totale sera aménagée, car le marché n'est pas assez grand pour que le projet se fasse en une seule phase.

\subsection{Propriétés du sol}

Le sol doit répondre à différents critères pour qu'on puisse l'aménager en espaces résidentiels: qu'on pense à la résistance, à sa stabilité et au drainage des eaux.

Les études géologiques nous ont appris que le sol était constitué d'argile ayant une grande capacité à la résistance 
au cisaillement. Ces résultats proviennent des échantillons de sol prélevés sur le terrain et analysés en laboratoire.

Concernant la stabilité du sol, le géologue doit analyser la morphologie et l'histoire de sa formation. On croit que lors du retrait de la mer de Champlain, les deux bords élevés du boulevard Université constituaient un seul plateau. La rivière Langevin a creusé son sillon avec le temps jusqu'a son lit d'aujourd'hui. Des glissements de terrains ont eu lieu auparavant. Il est difficile de mettre une date à ces événements. Nous avons entendu deux faits à ce sujet.

Le premier: lors de la construction du boulevard Université, on a trouvé un tronc d'arbre qui a été analysé au carbone 14. Ce tronc était âgé de 2000 ans.

Le deuxieme point provient des dires d'une religieuse. Lorsque les premières soeurs hospitalières arrivèrent dans la région, elles apprirent des autochtones qu'il y avait eu des glissements de terrains dans cette zone au 15e siècle.

Pour le drainage, étant donné que le sol est à flanc d'une vallée, l'écoulement des eaux se fait facilement vers le bas et elles se jettent dans la rivière à quelques centaines de mètres plus loin.

La surface du sol est relativement peu accidentée, mais un travail de nivellement est nécessaire sur la moitié de la superficie. Une coulée traversant en largeur le terrain devra être canalisée et remblayée. 


\subsection{Services d'égout, aqueduc et électricité}

Les services sont à proximité du terrain. Des réalisations en aménagement des eaux usées ont été faites sur le terrain et vont aider au développement de ces infrastructures.

Aucun problème ne se pose pour le raccordement à ces services.

\subsection{Coût du terrain}

Afin de pouvoir faire des comparaisons, une visite au bureau d'enregistrement a permis de connaitre le prix des derniers achats de terrains à des fins de lotissement.

Notre prix demandé, légèrement supérieur à la moyenne des dernières transactions, fut considéré raisonnable, compte tenu du site.

Nous savions que son coût d'aménagement serait semblable à la concurrence. Nous avons effectué une étude "pro forma" de la rentabilité du projet, étude que l'on retrouve dans le premier chapitre.

I1 est difficile de déterminer précisément le bénéfice net du projet. Cette étude nous démontra que l'aménagement du sol en espaces résidentiels n'entrainait pas de problème hors de l'ordinaire: le coût de l'aménagement se situerait dans la moyenne pour un sol d'argile.

L'étude de la concurrence nous révéla que les projets de "beaux quartiers" se situaient sur le roc. Le coût d'aménagement dans le roc est le double de celui de l'ar- 
gile. Tout en sachant que le prix d'achat du "terrain brut" dans le roc est moins élevé que pour de l'argile, ce point augmentait notre confiance dans la rentabilité du projet. 


\section{CHAPITRE 4}

\section{ANALYSE DE IA PROBLEMATIQUE}

Nous discuterons des principaux problèmes à affronter et à résoudre pour conduire le projet jusqu'à sa réalisation.

\subsection{L'organisation}

L'entreprise est constituee de deux partenaires. Ce n'est pas leur activite principale, mais chacun y consacre une dizaine d'heures par semaine. Une secrétaire s'occupe de la comptabilité et la correspondance. Cette personne participe souvent aux discussions et donne son point de vue sur la bonne marche de l'entreprise.

Dans cette equipe, on retrouve une personne expérimenté en promotion. EIle a une forte experience dans les secteurs hospitaliers et comerciaux. Son expertise acquise par le passé permet de guider l'entreprise avec maîtrise. Les portes s'ouvrent facilement devant elle.

Ce projet demande de s'allier de bonnes personnes ressources: entrepreneur, ingénieur, arpenteur... Ce choix permet d'arriver à de meilleurs résultats et plus rapidement si votre intervenant est le mieux placé auprès des autorités pour defendre votre point de vue. Il est très important de trouver la bonne personne ressource. Ce choix demande reflexion et surtout connaissance des personnes du milieu. 
Nous désirions faire affaire avec les gens les plus habiles à conduire notre projet vers la réussite. Nous devons nous informer pour bien connaitre la dynamique du milieu afin de faire les bons choix. Cet associé détient l'information pour effectuer les bons choix.

L'autre personne constituant l'équipe est débutante en promotion immobilière. A son grand désir d'apprendre s'ajoute une forte motivation pour réussir le projet. Le fait d'être associée à une personne expérimentée rend $\mathrm{sa}_{\mathrm{z}}$ tâche beaucoup plus facile et constitue un apprentissage du secteur des affaires fort aisé. Cette association est une chance inouïe pour le nouveau venu.

Les prises de décision sont basées sur l'entente mutuelle: toute décision est précédée d'une période de réflexion. Bien souvent on ne retrouve qu'une possibilité de solution. Lorsqu'il y a mésentente, la discussion est reprise, parfois laissée en suspens, pour $y$ revenir et arriver a une entente. Ce cheminement s'est fait sans peine jusqu'à maintenant. 
MODELE DE PROCESSUS DÉCISIONNEL

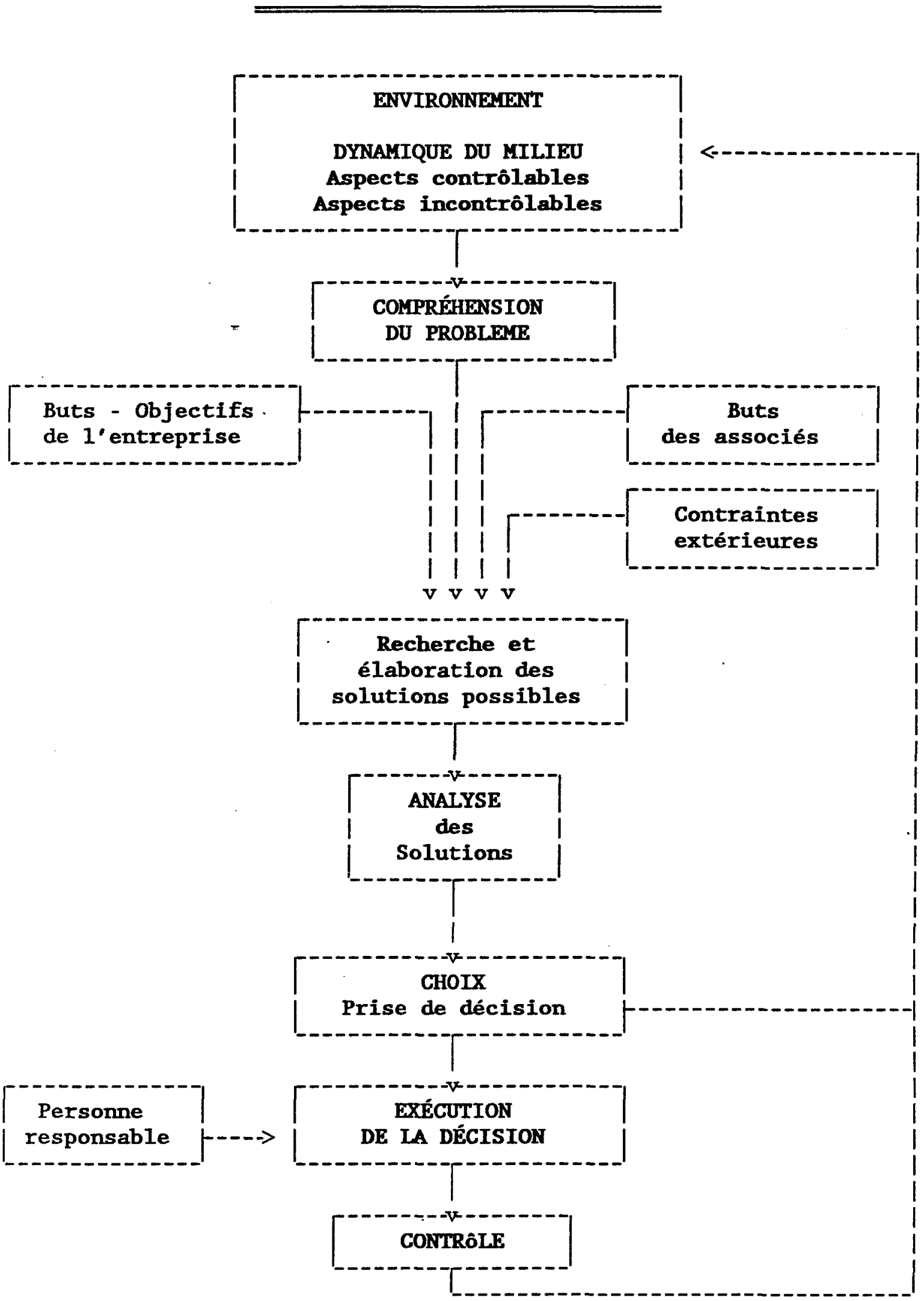




\subsubsection{Structure organisationnelle}

Une compagnie privée a été formée selon la loi provinciale. Le capital des actions a été réparti en deux parties égales entre chacun des actionnaires.

Cette proportion demande l'entente mutuelle pour la bonne marche de l'entreprise. La collaboration est essentielle et devient une association indivise en quelque sorte. Ceci crée un climat de confiance entre les partenaires.

Le fait d'être deux personnes permet les avantages de l'association sans subir les désavantages du groupe. Le champ où 1'on retrouve principalement cette importance est dans la négociation. Ce domaine constitue un facteur essentiel pour la réussite de l'entreprise: nous devons minimiser les possibilités d'erreurs de négociation.

Etre deux pour s'acquitter de cette tâche donne à l'entreprise un potentiel accru. Un associé peut négocier individuellement sans prendre de position formelle à la fin. La réunion des deux personnes dans une négociation donne souvent plus d'impact et de chances de réussite.

\subsubsection{Structure financière}

Le financement de l'entreprise fut fait par crédit bancaire garanti. Chaque personne est responsable de la moitié de la dette sans aucune responsabilité de l'autre partie envers la banque. 
Etant donné les ressources financières des deux associés, l'entreprise peut profiter d'un taux d'intérêt avantageux.

Les dépenses de l'organisation sont réduites au minimum. Les résidences de chacun servent comme bureaux. Le téléphone est le téléphone personnel. L'entreprise profite également des services d'une secrétaire sans aucun frais car un associé a déjà une telle personne-ressource pour ses affaires.

Le fait de n'avoir aucun frais fixe, sauf l'intérêt des prêts, est un point important pour la rentabilité du projet. Les dépenses sont réduites à leur minimum. Les partenaires de l'entreprise désiraient encourir le minimum d'inquiétude quant aux finances de l'entreprise. 


\subsection{L'environnement}

\subsubsection{L'environnement physique}

De nouveaux quartiers se développent continuellement. Ces quartiers offrent différents types d'environnement. Pour qu'un quartier projette une image de haut de gamme, il doit offrir un site avec des caractéristiques qu'on ne retrouve pas dans le quartier de "moyenne gamme".

Il va sans dire que l'environnement physique est l'élément de base pour structurer par la suite un lotissement résidentiel. Dans le chapitre 3 , nous avons énuméré les conditions préalables d'un bon site. Il est primordial d'attacher une grande importance à ce point.

Pour ce faire, 1'entreprise a engagé deux urbanistes pour déterminer un plan d'aménagement et établir une réglementation de construction.

Une visite de lotissements renommés d'autres villes a permis également de visualiser les points importants. Le lotisseur doit acheter le terrain et le transformer. Il doit produire des lots bâtissables. Cette transformation doit être basée sur une recherche. Les personnes qui peuvent contribuer à cette recherche sont bien sûr les urbanistes, mais également l'arpenteur, 1'ingénieurconseil et le maitre d'oeuvre.

L'ardeur déployée pour la conception du lotissement est la source du succès du projet, ceci constituant la motivation de la compagnie. 
Une conception banale donnera un quartier banal tandis qu'une conception soignée maximisera les chances de produire un quartier réussi.

Une règle d'or en immobilier: "site idéal, produit idéal et prix idéal." Le promoteur doit choisir un bon site, le lotir selon les principes d'urbanisme et a un prix raisonnable.

\subsubsection{L'environnement économique}

L'achat de biens fonciers a toujours été considéré comme un placement. Ce point pourrait peut-être prendre de l'ampleur depuis le "krach boursier" d'octobre 87 . Cette baisse a diminué de beaucoup 1 'intérêt des épargnants.

De plus en plus, ces personnes ayant un bon revenu se demandent où 1 'investir. On redécouvre que la maison demeure un placement intéressant.

L'investissement suit une certaine mode. De 1985 à 1987, 1'intérêt des gens était pour la bourse. Il semble se dégager depuis ce temps un certain engouement vers l'immobilier. On redécouvre que le placement dans la résidence familiale est un investissement sûr, non imposable, tout en étant un bien essentiel où l'utilisateur jouit quotidiennement de son placement.

Tout le marché des résidences profiterait de cette mode, autant le bas de gamme que le haut de gamme, le prix reflétant le produit. 
Même si cette nouvelle ferveur ne se réalise pas, l'habitation demeure un besoin primaire relié à la qualité de la vie et sera toujours en demande. On cherche toujours a augmenter cette qualité de la vie. Les gens portent de plus en plus attention à l'alimentation, aux loisirs, à la santé, au logement et à bien d'autres produits.

Il est plus difficile de constater un tel fait dans un petit environnement. Mais lorsqu'on regarde dans les grandes villes, ce phénomène est plus probant. La demande pour les grandes maisons est de plus en plus forte, tandis que la petite maison diminue en popularité.

Dans notre région, le climat économique reflète une santé économique de degré moyen. La grande entreprise est en décroissance pour l'instant. L'optimisme en économie $n^{\prime}$ est pas partagé par toute la population. Ces craintes justifiées laissent les habitants de la région sur une certaine défensive. Trouvera-t-on des preneurs pour des maisons de cent cinquante mille dollars et plus? C'est la question que beaucoup de gens se posent.

Une réponse positive à cette question peut constituer un élément de marketing.

Présentement on retrouve à Chicoutimi quelques secteurs où l'on construit des maisons "haut de gamme". La mise en marché de l'entreprise va devoir déplacer ces clients vers un nouvel espace résidentiel, d'où l'importance de la qualité du produit. 
Les entrepreneurs groient que le climat économique présent et la qualité du produit sont des points favorisant ce déplacement de clientèle vers leur projet.

\subsubsection{L'environnement politique}

Un projet de lotissement domiciliaire doit être accepté par le Conseil de ville. Le promoteur doit soumettre le projet aux fonctionnaires, qui eux, le présentent pour acceptation par les élus.

Le promoteur paie tous les frais d'infrastructure du lotissement sauf le recouvrement bitumineux de la rue, les bordures et l'aménagement des parcs, s'il y a lieu. C'est le promoteur qui donne le terrain pour le parc; si la municipalité ne désire pas un parc, elle réclamera au promoteur $10 \%$ de la valeur du terrain en argent.

Avec de telles conditions à respecter, les entrepreneurs pensaient être bien accueillis des fonctionnaires municipaux.

L'acceptation du projet fut très difficile et a demandé beaucoup de temps et d'énergie. Malgré leur détermination, la victoire n'a jamais paru décisive. Deux problèmes ont été soulevés: 1a géologie, et le réaménagement d'une rue existante.

Le point de la géologie a demandé beaucoup de temps de négociation. Un climat de durcissement s'était créé entre les parties. 
Lorsqu'il y a eu entente sur ce point, les promoteurs pensaient que toutes les difficultés étaient abolies. Un autre obstacle fut soulevé, soit le détournement d'une rue pour relier le lotissement.

Cette rue projetée passait sur le lotissement et également sur un terrain d'une tierce personne; la négociation devenant tripartite, soit la municipalité, cette personne et notre entreprise. Un imbroglio s'ensuivit: nous ne pouvions pas faire démarrer le projet avant que la municipalité ne s'entende avec cette tierce personne. L'entreprise était coincée.

Les promoteurs se sont joints à un entrepreneur spécialisé dans la construction de rues et ayant une bonne relation avec les fonctionnaires municipaux. Cet entrepreneur a joué le rôle de "démarcheur" afin de négocier une entente. S'il a réussi à régler certains points en litige, par contre il était impuissant devant d'autres aspects de la négociation. Les promoteurs devaient s'occuper personnellement de cette tâche.

On conclut qu'il serait profitable d'amener cette négociation avec les fonctionnaires sur le plan politique, c'est-à-dire avec le comité d'urbanisme et le conseil municipal.

Un autre processus de négociation s'ensuivit avec des règles de forces différentes. Ceci permit de faire avancer la négociation et d'arriver à une solution négociée. 
Une dynamique de négociation s'était installée et n'était aucunement en notre faveur. Il n'y avait aucun rapport de force. Notre entrepreneur avait fait avancer la négociation, mais il se rendit compte qu'il était impossible pour lui de continuer devant certains obstacles. Nous devions régler nos problèmes.

Nous étions pris dans un processus où notre projet ne pouvait se réaliser étant donné que la ville n'avait pas trouvé une solution au problème. Nous devions les forcer à faire avancer notre projet. Nous étions décidés à embaucher un conseiller juridique et poursuivre une démarche pour débloquer la situation.

Nous avons informé les fonctionnaires municipaux de notre décision. Il ne fut pas nécessaire de la réaliser. Tout se régla dans une journée. Nous avions atteint le "bout du rouleau".

Ce moyen de persuasion fut extrêmement profitable. Il va de soi qu'il est préférable de ne pas 1'appliquer. C'est une procédure de dernier recours.

Nous continuons à mettre de la pression sur le conseil municipal. Nous les informons de nos difficultés. Nous avons demandé leur aide. Nous avons fait intervenir d'autres personnes auprès du maire pour appuyer notre demande. Ces démarches également nous ont grandement aidés. 


\subsection{Description du processus}

Dans cette partie du chapitre, nous élaborons la démarche qui a été poursuivie du début jusqu'à la mise en marché du projet. L'ordre des problèmes soulevés respecte la chronologie.

\subsubsection{L'achat du terrain}

Avant d'entamer les négociations, nous, les promoteurs, avons élaboré la stratégie que nous allions adopter. Dans un premier temps, nous avons relevé les dernières transactions enregistrées d'achat de terrain dans le but de lotir. Etant peu familiers avec la valeur à payer pour un tel achat, nous nous sommes basés sur la transaction la plus récente effectuée de la part du vendeur.

La différence de prix était très grande, oscillant entre $0,04 \$$ et $0,85 \$$ le pied carré. On retrouve deux facteurs importants influençant le prix:

\section{a) Qualité de sol}

Un sol rocheux est très dispendieux à aménager et se vend pour quelques sous. Un sol argileux ou de gravier de préférence est beaucoup plus facile à aménager, un tel sol se vendant beaucoup plus cher. Le coût des infrastructures est de $8000 \$$ à $12000 \$$ dans l'argile et est au moins le double dans le roc. 
b) Prix global ou net:

Le prix peut être basé sur l'ensemble du lotissement, incluant les rues et le parc, ou sur la superficie cadastrée. Pour l'achat global, on doit figurer $30 \%$ de la superficie pour les rues et 108 , règlement municipal, pour le parc.

Après avoir analysé ces facteurs, nous nous sommes entendus sur les conditions de paiement que nous allions offrir. La stratégie était de demander beaucoup pour avoir une bonne marge de négociation.

Lors de la première rencontre, nous avons offert un prix au pied carré qui sera cadastré et demandions un financement complet de la part du vendeur jusqu'au moment de la vente a un tierce, financement portant intérêt à 98 sur la période encourue après quatre-vingt-dix jours de la date d'achat.

Cette offre permettait d'effectuer un achat par phases. On ne cadastrerait que les terrains qu'on espérait vendre par année.

Nous avons joint à cette offre écrite deux chèques de $25000 \$$ chacun pour démontrer du sérieux.

Nous avons présenté cette offre au conseil d'administration $d u$ vendeur. Les membres de ce conseil ont majoré les conditions de paiement, le taux d'intérêt, l'échéance de l'achat global et les condi- 
tions de garantie de paiement. Lors de cette rencontre, on a discuté de tous ces points, et ces conditions de l'entente allaient devenir définitives. Cette négociation fut brève et très importante. L'expérience de négociateur est un facteur essentiel dans une telle situation.

Les promoteurs apprenaient également du vendeur qu'un rapport de géologue avait été fait en 1984 et ne recommandait pas ce terrain pour 1'aménagement en quartier résidentiel. Le rapport pouvait être consulté mais sa diffusion était interdite.

Il a été convenu que le terrain devait être considéré comme bon pour le lotissement résidentiel, sinon l'offre d'achat n'était pas valide.

\subsubsection{La géologie}

Un géologue avait écrit un rapport sur le terrain convoité, recommandant de ne pas aménager ce secteur étant donné des possibilités de glissement de terrain.

Deux possibilités s'offraient à nous pour résoudre ce problème. La première était de demander à cet "expert" d'approfondir le sujet et de trouver des solutions d'aménagement du terrain. Nous avons discuté à quelques reprises avec lui et une telle avenue pouvait être envisagée.

La seconde était d'engager un autre géologue pour effectuer une nouvelle étude. Cette dernière 
possibilité fut retenue. Car elle permettait de mieux saisir la réalité. Si nous avions adopté la première solution, nous aurions été dépendants de cette personne; tandis que l'autre possibilité donnait le choix d'engager le géologue que nous penserions le plus qualifié.

Nous nous sommes informés auprès d'évaluateurs et d'ingénieurs du milieu pour connaître les meilleurs bureaux de géologues. Au début, nous pensions à Lavalin étant donné leur prestige. Le choix final se porta sur le groupe d'ingénieurs Roche, troisième en importance au Québec.

Dans notre région, il est toujours délicat de s'adresser à des professionnels de l'extérieur pour résoudre des problèmes du milieu. Cette situation peut provoquer un certain climat d'appréhension de la part du milieu professionnel régional, d'où l'importance d'embaucher un bureau de professionnels très réputé.

Un rapport concluant positivement à la possibilité de lotir le terrain fut présenté aux fonctionnaires municipaux.

Une contre-expertise fut demandée par le Service technique de la ville. Des échanges écrits s'effectuèrent entre les deux géologues. Ces échanges de points de vue menaient à des impasses. La géologie n'est pas une science exacte. L'idée d'un géologue vaut celle de l'autre. Le déroulement du débat devient sans issue.

Pour aider l'expert, nous avons consulté un autre bureau de la région qui avait fait des 
forages auparavant pour une société gouvernementale sur notre terrain. Leur idée du terrain était la même que celle de notre expert. Ce dernier put se servir de ces propos pour justifier ses dires. A deux contre un "l'adversaire" finit par fléchir et déclara qu'il était en désaccord avec la conclusion du rapport, mais qu'à son avis, 1'ingénieur avait effectué une étude sérieuse.

Ce débat n'a pas fait plus de lumière sur la question scientifique.

\subsubsection{Le plan d'aménagement}

Tout en discutant des propriétés du sol, nous devions préparer le plan d'aménagement, c'est-àdire le dessin des rues, et déterminer le zonage pour les différences de densité d'habitation. Le lotisseur jouit d'une certaine liberté concernant l'aménagement. Nous avons consulté deux urbanistes et un bureau d'arpenteurs pour faire le plan.

Le plan fut présenté au Service technique et au Service d'urbanisme de la ville. Lors de cette rencontre aucun problème majeur ne fut soulevé, sauf qu'une partie d'une rue d'accès devait être modifiée. On nous informa que la ville était en négociation avec le propriétaire du terrain pour réaménager cette rue. On nous demandait un délai de deux semaines pour résoudre ce problème. Le différend entre géologues n'étant pas terminé, il $n^{\prime} y$ avait aucun inconvénient à attendre.

Les fonctionnaires avaient le choix de négocier ou d'exproprier pour résoudre le problème de déviation de la rue. Ils choisirent la négocia- 
tion. Nous ne voulions aucunement être mêlés au débat. On nous demanda au début de régler ce problème. Nous avons expliqué que ce problème datait d'une vingtaine d'années, que la ville ne l'avait pas réglé et que nous ne pouvions pas faire ce travail car nous n'avions aucune force de négociation.

La tâche de négocier ne pouvait être assumée que par les représentants de la ville, soit les fonctionnaires ou les élus. Les cadres se sont attaqués à cette négociation.

Ces derniers nous firent plusieurs propositions. On désirait que nous versions à la ville une partie des frais d'aménagement du terrain qui était la propriété d'une tierce personne. Nous nous opposámes à cette demande et à toutes celles dans le même sens que nous trouvions saugrenues.

Nous avons examiné les titres de propriété du terrain. Nous avons découvert que la ville avait accepté devant notaire un plan où l'accès de la rue était bien délimité. On présenta ce plan aux fonctionnaires. Ces derniers prétendirent que ce document avait peu de valeur, qu'ils avaient le pouvoir discrétionnaire de refuser la permission de lotir.

Les relations devenaient de plus en plus tendues. Nous désirions être de tempérament conciliant. Mon associé disait que nous devions prendre une attitude plus agressive.

Nous avons engagé l'entrepreneur pour effectuer les travaux d'infrastructure. Notre choix 
se porta sur l'entrepreneur qui avait de bonnes relations avec ces fonctionnaires. L'entrepreneur nous conseillait dans nos démarches avec les fonctionnaires. C'est lui qui prétendait qu'on devait être conciliants. Le printemps était commencé. Nous n'avions pas la permission d'effectuer les travaux. Nous décidions de nous montrer très fermes.

Nous fimes des représentations au Conseil municipal. Nous rencontråmes les élus qui siègent au Comité d'urbanisme. Nous leur avons expliqué le problème, les fonctionnaires étaient présents. Un meilleur climat de négociation apparut. On nous fit une proposition acceptable qui reliait les deux parties: la ville construirait la rue a condition qu'on leur cède les terrains de chaque côté de cette rue, proposition qui fut acceptée.

C'était la mi-juin. La ville nous permettait d'effectuer le lotissement. La bonne période des ventes de terrains était passée, soit le printemps. Les lots ne pourraient être prêts à bâtir qu'au début d'août. Nous décidâmes malgré tout de d'entreprendre le projet. Les plans de génie avaient été acceptés aux différents niveaux de décision.

\subsubsection{Le plan de financement}

Une structure de financement avait été élaborée depuis le début du projet. Nous devions maintenant trouver une banque pour fournir les fonds nécessaires.

Le principe de base était d'utiliser l'emprunt d'argent. Nous désirions un financement complet. Nous avons vu auparavant qu'une compa- 
gnie privée fut fondée et était détenue à parts égales par les deux associés. Cette compagnie, possédant peu d'avoirs, ne suffisait pas pour effectuer l'emprunt nécessaire: chaque associé dut garantir la moitié de la somme, de façon divise et non responsable de l'autre partie.

Le financement bancaire fut accepté sans difficulté.

Dans ce chapitre, nous avons voulu décrire les problèmes de fond que les promoteurs ont rencontrés pour lancer le projet. II était indéniable qu'ils devaient résoudre chacun de ces problèmes pour faire démarrer l'entreprise. On savait, au début, qu'ils auraient des obstacles à surmonter. La ténacité était nécessaire pour réussir.

Il fallait croire à la réussite car à certains moments, elle n'était aucunement évidente. Il ne fallait pas se décourager, mais plutôt mettre plus d'ardeur à comprendre la situation. La réflexion, c'est les trois quarts du travail. Il faut connaître les forces et les faiblesses constituant le litige pour trouver une solution efficace et rapide.

Le choix de démontrer un pouvoir de négociation nous a bien servis. Au début des négociations avec les fonctionnaires municipaux, ce pouvoir ne fut pas exprimé. Ces derniers disaient qu'ils avaient pleine autorité. Les déblocages se sont faits lorsque les promoteurs démontrèrent un pouvoir en effectuant des pressions sur les élus municipaux et les fonctionnaires. Nous avons brandi la menace de poursuivre devant les tribunaux si une décision satisfaisante n'était pas rendue dans un délai raisonnable.

Ce jeu de force exigeait une attitude de "puissance" pour arriver à une conclusion. 


\section{CHAPITRE 5}

\section{IES PROBLEMES SECONDAIRES}

Nous avons traité dans le chapitre précédent des obstacles à résoudre pour pouvoir mettre en marche le projet. C'étaient des problèmes majeurs qui demandaient une solution pour permettre la mise en chantier du projet. Dans ce chapitre, nous discuterons des questions permettant une valorisation du projet, c'est-à-dire comment fournir un meilleur produit et comment le faire connaitre.

\subsection{Un meilleur produit}

Le but est de créer un beau quartier. Le travail est de bien planifier les variables pour permettre d'arriver à ce but. Les associés voulaient n'être que lotisseurs et non pas constructeurs. Il est certain que celui qui effectue tout le travail, arrive à batir un quartier selon ses visions. Ne désirant aucunement être constructeurs, nous devions rédiger une réglementation concernant la construction afin de garder une certaine harmonie entre les bâtiments.

On devait agir sur le dessin des rues et sur le contrôle de la construction. 


\subsubsection{Le dessin des rues}

La forme de lotissement en quadrilatère est la plus répandue dans nos villes. Ce modèle est peu esthétique. Nous préférions un lotissement avec des lignes courbes plutôt que droites, le terrain se prêtant très bien à cette idée. Nous avions observé dans la région que les beaux quartiers respectaient ce principe, qu'on pense à certains quartiers d'Arvida, Riverbend et Isle-Maligne à Alma, Notre-Dame-du-Saguenay à Chicoutimi.

Ce modèle est de plus en plus appliqué dans les municipalités où l'urbanisme est développé. Certaines critiques diront qu'il est plus dispendieux d'utiliser ce modèle, mais la différence de coûts n'est pas si marquée. Des ouvrages sérieux en urbanisme ont fait la comparaison, et dans certains cas, ce modele peut entraîner une diminution de longueur de rues, donc un coût moindre pour le même espace résidentiel.

La courbe offre deux grands avantages: premièrement, elle donne un meilleur aspect visuel; deuxièmement, la vitesse de circulation automobile est ralentie. Ce modèle démontre généralement un travail d'urbanisme plus soigné.

On demanda aux urbanistes et à l'arpenteur d'essayer d'utiliser ce modèle dans le dessin des rues. 
5.1 .2 Le parc

Un règlement municipal oblige de céder 108 de l'espace résidentiel à la ville pour l'aménagement d'un parc ou bien de verser la valeur de cet espace dans les coffres municipaux. Un espace vert étant un ajout d'embellissement, on opta pour ce choix. Les urbanistes et l'arpenteur ont fourni des possibilités de situation. On choisit un site central, bien en vue, permettant un dégagement visuel.

\subsubsection{La réglementation}

Le coup d'oeil que présente toute une rue dépend de $I^{\prime}$ apparence collective. C'est pourquoi il est important de dicter des idées directrices pour créer une harmonie visuelle collective.

Nous avions des idées de réglementation que nous avons soumises aux urbanistes pour qu'ils corrigent et rédigent une formulation appropriée. Nous désirions une réglementation accordant une certaine liberté aux résidents tout en interdisant les projets qui pourraient dévaloriser le plan d'ensemble. Nous voulions donner aux résidents une certaine liberté de conception. Nous n'étions aucunement d'accord que tout soit réglementé comme cela existe dans un nouveau lotissement à Chicoutimi. On voulait se situer à mi-chemin entre ce concept et l'anarchie opposée. (Voir annexe VII).

L'étude de marché avait démontré que $1^{\prime}$ idée de réglementation devrait être souple tout en déterminant des limites bien sûres. La réglementation 
est une sorte d'assurance pour les premiers rési-. dents du quartier.

Ces parametres permettent une projection de développement du quartier. La construction d'une résidence nécessite des fonds importants. Si on laisse au hasard 1'harmonie, les premiers résidents prennent un risque car ils ne savent pas l'image qu'aura le lotissement, tandis qu'une réglementation minimise le risque. Ce point devient un élément de marketing. Tous les acheteurs sont en accord avec les règlements car cela protège leur investissement.

Sans règlement, on ne peut avoir une idée du lotissement futur, l'harmonie visuelle est laissée au hasard. Un bâtiment de mauvaise apparence peut être désastreux pour l'entourage. Pour contrer cela, chaque projet devrait être présenté et accepté avant construction. En cas de doute, les promoteurs pourront demander l'opinion d'architectes ou d'urbanistes.

\subsubsection{L'application des règlements}

Deux possibilités s'offraient pour la méthode d'application des règlements:

a) faire adopter la réglementation au niveau municipal: cette méthode permettait un contrôle externe, très rigide, hors de la portée des promoteurs. 
b) I'annexer au contrat d'achat: les règlements deviennent des servitudes.

Cette dernière solution fut retenue car elle permettait de garder le contrôle de la réglementation. Il était possible d'effectuer des dérogations mineures sans enclencher un processus complexe. La réglementation, devenant un acte notarié, permet d'exercer un droit de recours envers l'acheteur ne s'y conformant pas.

Il est peu probable qu'une personne agisse de mauvaise foi et déroge de façon très marquante de la réglementation.

\subsubsection{L'enfouissement des fils électriques}

La disparition des fils électriques et des poteaux contribue à augmenter la qualité visuelle d'un espace résidentiel.

Les promoteurs étaient déterminés au début à appliquer cette méthode. Le coût supplémentaire pour le faire nous a fait changer d'idée. HydroQuébec défraie entièrement les coûts du réseau aérien, mais la différence de coûts pour le réseau souterrain par rapport à l'autre est à la charge du lotisseur. Le coût du réseau souterrain est dix fois plus élevé. Le coût de l'implantation d'un tel réseau augmenterait de $4000 \$$ environ le prix de chaque terrain. Nous ne pensions pas qu'un propriétaire de terrain était favorable à débourser 
une telle somme pour ne plus voir de poteaux et de fils.

Effectuer une telle dépense aurait pu mettre en péril le projet.

\subsubsection{Mobilier}

Deux idées furent explorées concernant le mobilier urbain. La première était l'installation d'un petit lampadaire à chaque entrée privée. Cette idée fut refusée par le service municipal d'urbanisme. La raison évoquée fut que le fait de répéter le même modèle de lampadaire crée une habitude visuelle qui tend à l'oublier, à le faire disparaitre.

La deuxième idée était de mettre un accent sur les deux entrées du lotissement. Nous pensions à la fabrication de deux piliers, un de chaque côté de la rue, qui constitueraient une porte de quartier comme on en voit à l'entrée d'un domaine ou de certains quartiers anglo-saxons.

Une autre idée fut finalement retenue, soit d'utiliser un monument au centre de la rue au lieu d'une porte. Elle fut soumise et acceptée au service d'urbanisme. de la ville. L'idée a été transmise à l'arpenteur et à la firme d'ingénieurs pour faire les plans en conséquence. Dès que le budget va le permettre, les promoteurs érigeront un premier monument. Ce point va dénoter, si c'est bien conçu et bien fait, un certain prestige. Ceci est un désir depuis le début de l'entreprise. 
Les promoteurs espèrent que cette idée démontrera le travail qu'ils ont essayé de faire pour que le lotissement soit réussi.

\subsection{Le marketing}

\subsubsection{Le produit}

Nous avons discuté, jusqu'à cette étape du mémoire, des caractéristiques physiques du produit que l'entreprise désire offrir a un segment de marché que le lecteur peut entrevoir. Mais comme les "marketers" l'affirment, "le produit est bien plus qu'un ensemble de caractéristiques physiques: le produit est constitué de la promesse de satisfaction faite par l'entreprise a tous ses clients potentiels".1

Notre produit qui demande un prix élevé exige une promesse de satisfaction crédible. On doit faire connaitre aux clients:

A - les caractéristiques physiques du terrain;

B - la présence d'une réglementation concernant la construction, qui constitue une sorte de garantie du produit;

C - un nom commercial qui projette une image de prestige.

1 Le Marketing, fondements d'applications. R.Y. Darman, M.Laroche, J.V. Petrof. (p. 177) 1978, McGraw-Hill 
Le choix d'un nom demande une certaine réflexion. Le premier choix était "La Seigneurie des Augustines". Nous trouvions que "Seigneurie" faisait prestigieux et "Augustines" était le nom de la communauté religieuse à qui appartenait le terrain. Ces dernières refusèrent ce nom. Un autre nom fut retenu pour la désignation du lotissement: "La Seigneurie des Lilas". On inclut dans la réglementation qu'on devait retrouver un lilas devant chaque habitation.

\subsubsection{Le positionnement du produit}

On désirait que le produit devienne le nouveau quartier "chic" de la ville. Trois autres lotissements (Domaine du Roy, Panoramique, Quartier modèle) se disputaient ce titre, chacun ayant des caractéristiques propres. Leur produit étant nouveau, on devait créer la réputation. On misa surtout sur un point, celui du contrôle de la construction. Le fait d'intererdire certains matériaux de revêtement extérieur donnait une relative apparence de "snobisme". On n'était aucunement frustré d'avoir cette réputation.

Il fallait prendre la place de leader. On devait présenter le produit comme étant inévitablement le meilleur. On devait percer le marché le plus rapidement possible. La publicité devait être rentable. 


\subsubsection{La publicité}

Le premier moyen publicitaire fut un kiosque a une exposition soit le "Salon de 1'Habitation 88". Ceci permit de rencontrer les personnes en "tête-àtête". Ce premier contact permettait de roder nos propos, de vérifier l'accueil du produit sur le marché, et bien entendu de lancer le produit.

Le fait de rencontrer de quatre à cinq cents personnes individuellement en cing jours fut une publicité qui demanda beaucoup d'énergie mais se révéla rentable.

Les réactions obtenues des gens furent très satisfaisantes. L'optimisme régnait. Nous étions convaincus par notre idée. I'intérêt de la population était grand. On "accrochait" le segment de clientèle désirée. Ce n'était pas tous les gens qui étaient intéressés. Le nombre de personnes constituant le segment était important dans cette activité publicitaire.

On avait apporté un grand soin dans la présentation du kiosque. Ce détail était très important pour obtenir un bon résultat.

Le deuxième moyen publicitaire fut d'ériger deux panneaux à chaque extrémité du terrain, annonçant le développement et les numéros de téléphone pour recevoir de l'information. On a également cherché un certain cachet, un "design", dans la fabrication de ces panneaux. 
Le troisieme moyen consista en des articles dans les journaux locaux (Le Quotidien, L'Extra). Deux articles ont porté sur le projet. Nous avons l'intention de renouveler les articles de temps à autre, surtout au début du printemps et à l'automne, périodes très propices à la vente de terrains.

Les concurrents font très peu de publicité. Ils utilisent des panneaux sur le terrain et les annonces classées dans les journaux.

\subsubsection{Leprix}

La détermination du prix demande une certaine réflexion. On doit tenir compte du prix de revient et de la concurrence pour établir l'échelle des prix.

Dans la plupart des cas, le pied carré est l'étalon de base pour fixer le prix. On demande tel montant par pied carré. Cette norme était difficilement applicable dans notre cas, étant donné que la superficie des terrains était plus grande que chez la concurrence. Nous établîmes, au début de la vente, un prix unitaire basé sur le coût de revient et la surface. Il a fallu réviser cette idée car elle était mal perçue de la clientèle. Nous sommes revenus avec un prix basé sur le pied carré tout en accordant une diminution de prix pour les terrains de grande surface.

Ce manque d'expérience s'est fait beaucoup sentir au début dans la négociation des prix. Par la suite, nous avons réussi à déterminer une échelle de prix réaliste et concurrentielle. Au début, 
les prix demandés furent moins élevés de façon à pénétrer le marché le plus rapidement possible; et au fur et à mesure que les ventes augmentaient nous avons relevé les prix, stratégie habituelle en affaires.

\subsubsection{La force de vente}

L'image de I'entreprise doit transparaitre également dans cet aspect du marketing. Le vendeur doit avoir une "tête" qui correspond au produit. Deux possibilités ont été discutées à ce sujet. La première étalt l'embauche de certains courtiers en immeubles comme vendeurs. La seconde était d'effectuer nous-mêmes ce travail.

On opta pour la seconde proposition pour différentes raisons: aucune charge monétaire, meilleure connaissance du produit, bonne disponibilité, meilleur "feedback" des clients,...

La place d'affaire était la résidence des promoteurs. On croyait que le décor de ces lieux devenait un argument de vente car le terrain de ces résidences est bien situé. La maxime du "cordonnier mal chaussé" ne s'appliquait aucunement dans ce cas!

On chercha également à bien accueillir les gens. Le fait de les recevoir au domicile facilitait cet aspect. Nous essayions le plus souvent d'être réunis pour rencontrer le client. Les gens ont des préférences: certaines personnes choisiront de continuer les négociations avec tel partenaire plutôt que 1'autre. Et si par hasard il y 
a mésentente, ils ont la possibilité de communiquer avec l'autre pour continuer la négociation. On augmentait les chances de vente.

On cherche également à entretenir un climat de négociation détendu, agréable, afin de développer une bonne relation avec les gens.

Cette méthode de vente fonctionne, et démontre bien le caractère de l'entreprise. Nous cherchons a bien faire comprendre les buts recherchés au client. Le début des ventes est plus difficile étant donné que le nombre d'habitations est peu élevé. Le client doit vraiment nous faire confiance.

Dès que le nombre augmente, le produit est plus concret. La vente devient une opération plus facile. 


\title{
CHAPITRE 6
}

\section{MODELE CONCEPTUEL}

\begin{abstract}
Nous présenterons la conceptualisation de la démarche à suivre pour la planification et la réalisation d'un lotissement résidentiel. Il s'agit d'un modèle de référence qui peut servir de guide pour explorer la réalité. Dans chaque projet, une ou des fonctions auront plus d'importance, mais cette démarche doit être effectuée pour arriver à nos fins. Certaines personnes sont devenus expertes dans la réalisation de cette démarche.
\end{abstract}




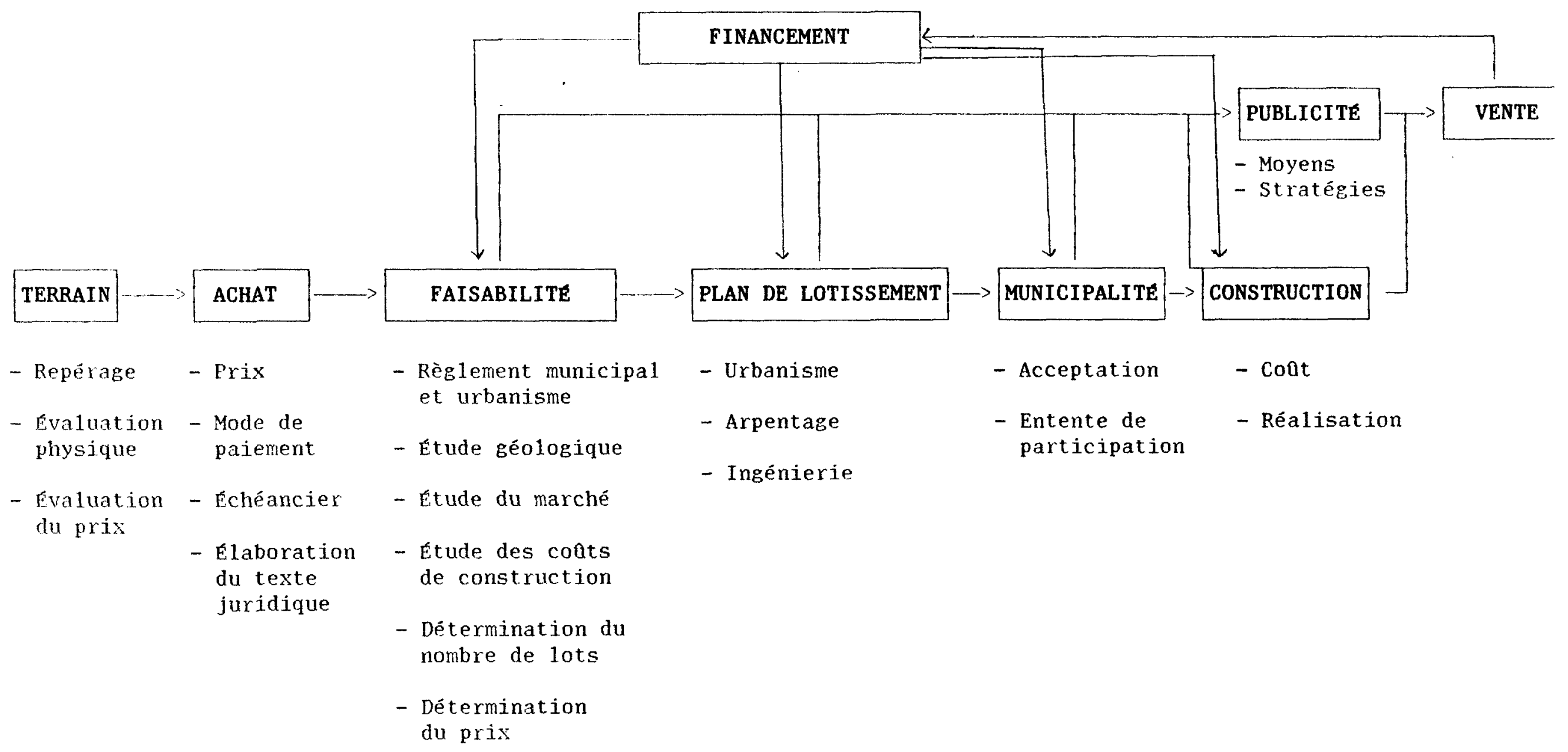




\subsection{APPLICATION DU MODELE}

Ce modèle, que nous voulions simple, parait peut-être pour le lecteur une démarche longue à appliquer. Nous voudrions "démythifier" chacune des étapes du modèle pour démonter sa simplicité.

\subsubsection{Terrain}

Repérer le terrain peut se faire par différents moyens: déplacement pédestre, photo aérienne, observation aérienne, etc.

L'évaluation physique du terrain demande un jugement sur les qualités du sol, de l'information sur les infrastructures environnantes (information qu'on recueille au Service technique de la municipalité), et un jugement sur le potentiel de vente des lots sur ce terrain.

L'estimation de la valeur du terrain demande de l'expérience, car l'activité de lotissement se déroule toujours de la même manière. Elle peut être accomplie par une seule personne ou quelques-unes dans un simple bureau car les promoteurs sont, en fait, des entremetteurs.

La tâche est de: repérer des emplacements pour développer de nouveaux espaces résidentiels; acheter le terrain; préparer une esquisse du projet; obtenir les permis nécessaires des autorités; trouver les prêteurs afin d'obtenir les fonds nécessaires; préparer les plans de lotissement et d'ingénierie; engager les entrepreneurs pour construire les rues; et finalement, s'occuper de la vente des lots. 
Nous pouvons utiliser les méthodes d'évaluation dans le domaine immobilier ou faire évaluer le terrain par un expert. Cette personne doit être très compétente.

\subsubsection{Achat}

Cette étape fait appel à 1'habilité de négociation et à la clairvoyance. Nous devons déterminer un prix juste, un mode et un échéancier de paiement en fonction de notre pouvoir de financement. Nous devons également prévoir des "portes de sortie" si le projet ne se réalise pas. Si tout cela est impossible à négocier, nous devons passer à l'étape de faisabilité avant de procéder à l'achat, de façon à minimiser les risques. Il serait important que le processus avec la municipalité soit enclenché et se déroule bien avant d'acheter.

\subsubsection{Faisabilité}

Le rôle d'entremetteur" débute dans cette étape. On doit réaliser $I^{\prime}$ asșemblage du montage. Le début des travaux avec les professionnels s'enclenche. On doit préparer avec eux une ébauche du projet pour permettre de recueillir toute l'information nécessaire à la prise de décision finale.

\footnotetext{
Certains points devront être résolus définitivement lors de cette étape, soit la géologie et le potentiel de rentabilité.
}

L'étape de faisabilité est en relation avec l'étape précédente. Le prix d'achat du terrain sera en fonction du coût de son aménagement et du prix de vente 
estimé, d'où 1'importance d'avoir une entente négociée permettant une certaine forme de réajustement.

\subsubsection{Plan de lotissement}

Cette étape est véritablement une táche de coordination. Il s'agit d'unir les idées valables de chaque intervenant: urbaniste, arpenteur, ingénieur et constructeur, afin de bien planifier la réalisation de l'espace résidentiel. On accordera de l'importance au dessin des rues, au mobilier urbain et à la réglementation des aspects physiques des maisons.

Lorsque les plans de génie sont réalisés, nous pouvons établir le coût de construction avec l'entrepreneur. Il est préférable de consulter quelques entreprises spécialisées dans ce domaine pour permettre d'avoir un prix avantageux. Nous devons considérer le prix et la compétence du constructeur pour effectuer un bon choix. Faire affaire avec des gens de commerce agréable enlève beaucoup de tracas.

\subsubsection{Municipalité}

Nous avons affaire aux fonctionnaires et aux élus. Le projet doit être accepté par les fonctionnaires et entériné par le conseil municipal.

La proportion de la participation financière "promoteur - ville" est de 80:20. La municipalité a des coûts à assumer dans le lotissement. Certains travaux sont entièrement sous sa responsabilité, tels le recouvrement bitumeux, les bordures, l'aménagement des parcs; d'autres sont partagés avec le promoteur. Ce partage des coûts est régi par des lois. Il demeure 
toujours une certaine part d'interprétation de lois, d'où naft le besoin d'une négociation entre les deux parties. Ces fonctionnaires font bien sentir leur pouvoir, d'où l'importance pour un néophyte d'être conseillé dans ces dédales administratifs. L'ingénieur et le constructeur sont de bonnes personnesressources pour mener a bien cette tâche.

\subsubsection{Gonstruction}

Nous avons fait notre choix de la firme de construction. Le projet se réalise, une bonne part de la tâche de la promotion est faite.

La surveillance des travaux est effectuée par le bureau des ingénieurs-conseils. Cette étape est facile pour le promoteur et très agréable si tout se déroule comme prévu.

\subsubsection{Publicité}

Cette étape peut débuter dès que le projet prend forme et que l'on croit a sa réalisation. Nous ne devons par perdre de temps pour informer les gens du nouveau quartier qui se projette, afin de minimiser le temps de vente.

Il est bon de planifier une stratégie publicitaire pour chercher à se démarquer de ses concurrents. Cette tâche est très importante pour le succès de l'entreprise. 


\subsubsection{Vente}

Cette tâche peut être assumée par le promoteur ou par un courtier. Cette dernière solution diminue substantiellement les profits.

Le promoteur est généralement bien placé pour s'occuper de présenter le produit au client et de négocier un prix satisfaisant les deux parties. L'habileté de vendre est importante. On doit chercher à connaitre les goûts du client pour lui présenter le terrain répondant a ses attentes. Nous devons garder un climat serein et agréable.

La vente permet d'alimenter la dernière fonction, soit: le financement.

\subsubsection{Financement}

Cette fonction chapeaute la démarche. Avant d'entreprendre cette démarche, il est nécessaire d'avoir une idée de la façon dont on réglera ce problème.

Il va sans dire que lorsque les avoirs du promoteur sont importants, le financement est facile à obtenir, étant donné qu'il peut garantir le prêt par son capital.

Le problème est plus difficile lorsque le promoteur a une capacité financière restreinte. On doit dans ce cas effectuer un montage financier en utilisant les différentes formes de financement, comme nous 1 'avons décrit dans le chapitre 4. 
Le moyen ultime est le financement par le vendeur, les consultants, 1'entrepreneur. Cette solution est plus difficile à réaliser mais certains promoteurs réussissent ce tour de force.

La pré-vente est un autre moyen à explorer.

Tout promoteur cherche à faire un profit avec "l'argent des autres". C'est également la meilleure façon de minimiser le risque d'en perdre.

La promotion immobilière est une tâche comportant des risques. La prudence et la parcimonie doivent être appliquées pour maximiser les chances de réussite:

"On ne doit pas mettre la charrue avant les boeufs." 


\section{CONCLUSION}

Cette recherche-action est entreprise depuis vingt mois. La phase décrite dans ce mémoire se rapporte au douze premiers mois. Présentement, la réalisation est commencée depuis un an.

Nous pouvons analyser les résultats de l'étude par rapport à la réalité vécue dans ce début de réalisation. Nos prévisions sont-elles conformes à la réalité?

L'entreprise se développe comme prévu. Ce projet dont on croit qu'il prendra trois ans pour être terminé, est bien démarré. Trente-cinq lots cadastrés ont été développés, plus des deux tiers ont été vendus, une quinzaine de constructions s'érigent. La qualité des résidences est même supérieure aux attentes de départ. Tout laisse croire en ce moment que le projet sera une réussite.

Les promoteurs doivent veiller au bon déroulement pour ne pas commettre d'erreurs pouvant mettre en danger l'entreprise.

Comme toute bonne réalisation, on doit prendre le temps nécessaire pour un développement harmonieux. On ne peut précipiter les composantes conjecturales: le temps est nécessaire pour mettre en place ces composantes.

Notre segment de marché est restreint. Le rythme de développement est plus lent qu'un marché de masse. L'entreprise ne peut se comparer aux autres qui visent ces marchés. L'entreprise doit avoir ses propres objectifs de réalisation et se donner le temps de réalisation nécessaire. 
Les coûts projetés se sont révélés inférieurs à la réalité. Par contre, le prix de vente a pu être augmenté également par rapport aux prévisions. L'augmentation annuelle des prix est très importante dans ce domaine et permet d'espérer une meilleure marge bénéficiaire dans le futur.

Nous pouvons croire présentement que ce projet "La Seigneurie des Lilas" sera une réussite pour les prometteurs et surtout pour les personnes habitant dans ce nouvel espace résidentiel. 
SONDAGE SUR LES TENDANCES EN

HABITATION A CHICOUTIMI

Dans le cadre d'une étude des tendances en habitation pour un projet de fin d'études de maîtrise à l'U.Q.A.C., auriez-vous l'amabilité de prendre quelques minutes pour remplir ce questionnaire.

Nous vous assurons de la confidentialité des données recueillies. De ce fait, vous ne devez pas inscrire votre nom.

Nous vous remercions de votre collaboration. 
1- Combien de personnes habitent la maison?

2- Dans quelle catégorie d'áge se situe le chef de famille?

25 à 30 ans $\quad 40$ a 45 ans [

30 a 35 ans $[-]$

35 à 40 ans $[-]$

3- Combien d'enfants habitent la maison?

De ce nombre combien...

a) sont trop jeunes pour fréquenter l'école?

b) fréquentent l'école primaire

c) "

" secondaire

d) "

" le collège

e) "

" 1 'université

f) autres

$-1$

4- Est-ce la première maison dont vous êtes propriétaire?

Si non, combien de maisons avez-vous possédées auparavant?

5- Votre maison est-elle unifamiliale

ou bifamiliale 
6- Le revenu principal de la famille se situe dans cette catégorie (en milliers de dollars)
20 à 25
30 à 35
40 à 45
25 a 30
35 à 40
45 et plus
$[1$

7- Le revenu familial se situe dans cette catégorie (en milliers de dollars).

le même que la réponse précédente
30 à 40
50 à 60
70 à 80
40 à 50
II
60 à 70
80 et 90
90 et plus

8- Etes-vous un travailleur

- a votre compte

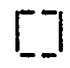

- salarié d'entreprise privée

- salariée gouvernemental public

para-public

- retraité

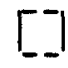

9- Dans combien d'années pensez-vous revendre la maison que vous habitez? 
10- Quels sont pour vous les points à prendre en considération lors du choix du quartier?

a) le prix du terrain

b) le prix des maisons de ce quartier

c) 1'aspect général du quartier

d) proximité du lieu de travail

e) proximité des écoles

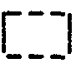

f) proximité des centres commerciaux

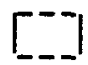

g) proximité des installations communautaires (terrains de jeux, tennis, piscine, etc.)

h) proximité des transports en commun

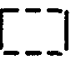

i) qualité de voisinage

j) autres...

11- Quel montant seriez-vous prêt à débourser pour l'acquisition d'une maison et du terrain?

12- Quel montant seriez-vous prêt à débourser pour un terrain seulement? 
13- Seriez-vous prêt à payer plus cher pour un terrain

a) près des centres comerciaux

oui non

b) près de 1'hôpital et de 1'université

oui non

c) plus grand que 70 pi. X 100 pi.

oui non

II

d) boisé

oui non

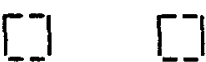

14- D'après vous, quelle grandeur devrait avoir un terrain en ville?

15- Pensez-vous qu'il serait important d'avoir des règlements lors de la construction des maisons pour avoir une harmonie générale?

oui non

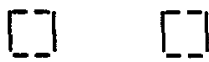

16- Ces règlements devraient-ils être très précis, ne laissant que peu de marges, pour s'assurer de l'harmonie en générale?

oui non

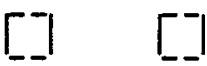

17- Seriez-vous pour réglementer

- le choix de couleurs des revêtements extérieurs oui non

- les matériaux de recouvrement extérieurs de la oui non maison

- I'architecture des maisons

oui non<smiles>C1CCC1</smiles>

- le temps pour faire l'aménagement paysager

oui non 


\section{ANNEXE II}

PROFIL DU RESIDENT: Quartier Alfred Boivin

1. Age: 30 a 35 ans 1008 ont entre 25 et 40 ans

2. Famille: 1 enfant

3. Revenu principal:

Revenu familial:

$40 \gtrsim 45000$

50 a 60000

$\$$

4. Emploi: a) à son compte

b) salarié d'entreprise

c) salarié gouvernemental

d) salarié para-gouvernemental

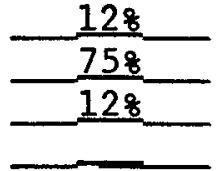

5. Prix prêt à payer pour un terrain: 21500 $\$$

6. Superficie de terrain souhaitée: $8100 \mathrm{pi} \underline{2}$

7. Prix prêt à payer pour la maison et le terrain: $120 \quad 000$ $\$$

8. Première maison qu'il possède: $12 \%$ Deuxième maison qu'il possède: $\underline{508}$ Troisième maison qu'il possède: $\underline{128}$ Quatrième maison qu'il possède: $\frac{128}{128}$ Huitième maison qu'il possède: $\underline{128}$

9. Points importants pour le choix du quartier:
A) Prix du terrain
B) Prix des maisons
C) Aspect général du quartier
D) Proximité du lieu de travail
E) Proximité des écoles
F) Proximité des centres commerciaux
G) Proximité des installations communautaires
H) Proximité des transports en commun
I) Qualité du voisinage

OUI \& NON8

\begin{tabular}{ll}
$-\underline{25}$ & -75 \\
$-\underline{25}$ & $-\underline{75}$ \\
$-\underline{25}$ & $-\underline{75}$ \\
$-\underline{25}$ & $-\underline{75}$ \\
$-\underline{37}$ & $-\underline{62}$ \\
$-\underline{0}$ & -100 \\
\hline
\end{tabular}


10. Serait-il prêt à débourser davantage pour un terrain?
A) Près des centres commerciaux
B) Près de l'hopital et de l'université
C) Plus grand que 70 pi $\times 100$ pi
D) Boisé

OUI \&

NON\&

$\frac{0}{12}-100$
-100
$-\underline{100}-\underline{0}$
$-\underline{0}$

11. Est-il favorable à une réglementation lors de la construction?

$\underline{100}+\underline{0}$

12. Est-il favorable à une réglementation très précise?

$\frac{37}{12}-\underline{50}$
$-\underline{12}$
$-\underline{25}$
$\underline{37}$

Nombre de répondants: 


\section{ANNEXE III}

PROFIL DU RESIDENT: $\quad$ Domaine du Roy

1. Age: 37 ans 808 ont entre 25 et 40 ans

2. Famille: 2 enfants

3. Revenu principal:

Revenu familial:

45000 et plus
70000 a 80000

4. Emploi: a) à son compte

b) salarié d'entreprise

c) salarié gouvernemental

d) salarié para-gouvernemental

$\begin{array}{r}\frac{408}{408}-\frac{208}{08}- \\ \hline\end{array}$

5. Prix prêt à payer pour un terrain:

$28 \quad 500$

$\$ 608$ entre 20

et 30000

6. Superficie de terrain souhaitée:

$8750 \mathrm{pi} \underline{2}$

7. Prix prêt à payer pour la maison et le terrain:

$150 \quad 000$ $\$$

8. Première maison $q u^{\prime} i l$ possède: $\underline{108}$

Deuxième maison qu'il possède: $\frac{108}{108}$

Troisième maison qu'il possède: $\underline{308}$

Quatrième

$\underline{408}$

9. Points importants pour le choix du quartier:
A) Prix du terrain
B) Prix des maisons
C) Aspect général du quartier
D) Proximité du lieu de travail
E) Proximité des écoles
F) Proximité des centres commerciaux
G) Proximité des installations communautaires
H) Proximité des transports en commun
I) Qualité du voisinage

OUI \&

NON8

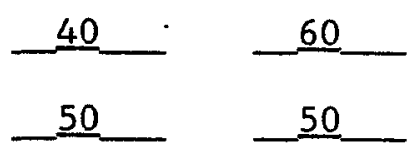

100

$\underline{50}$

군

2

$\underline{30}$

$-2$

70 
10. Serait-il prêt à débourser davantage pour un terrain?

$$
\text { OUI \& NON\& }
$$

A) Près des centres commerciaux

$\underline{0} \quad \underline{100}$

B) Près de 1'hopital et de l'université

$\underline{20}-\underline{80}$

C) Plus grand que 70 pi X 100 pi

90_ 10

D) Boisé

$\underline{90} \quad \underline{10}$

11. Est-il favorable à une réglementation lors de la construction?

$\underline{90}-\underline{10}$

12. Est-il favorable à une réglementation très précise?

$\underline{50}-\underline{50}$

13. Est-il favorable pour réglémenter?

A) les couleurs des revêtements extérieurs

$\underline{40}$

B) les matériaux de recouvrement extérieurs

$\underline{60}-\underline{40}$

C) I'architecture des maisons

$\underline{70}-\underline{30}$

D) le temps pour effectuer 1'aménagement paysager

$\underline{70}-\underline{30}$

Nombre de répondants: 10 


\section{ANNEXE IV}

PROFIL DU RESIDENT: Quartier Modèle

1. Age: 35 à 40 ans 848 entre 35 et 45 ans

2. Famille: 2 enfants

3. Revenu principal:

Revenu familial:

42000
42000

4. Emploi: a) à son compte

b) salarié d'entreprise

c) salarié gouvernemental

d) salarié para-gouvernemental

$-\frac{\frac{168}{338}}{\frac{338}{168}}$

5. Prix prêt à payer pour un terrain:

15000

33 z prêt à payer $20000 \$$

6. Superficie de terrain souhaitée:

$7000 \mathrm{pi} 2$

7. Prix prêt à payer pour la maison et le terrain:

$100 \quad 000$ $\$$

8. Première maison qu'il possède: 168

Deuxième maison qu'il possède: $\underline{508}$

Troisieme maison qu'il possède: $\frac{168}{168}$

Quatrième

$\underline{168}$

9. Points importants pour le choix du quartier:
A) Prix du terrain
B) Prix des maisons
C) Aspect général du quartier
D) Proximité du lieu de travail
E) Proximité des écoles
F) Proximité des centres commerciaux
G) Proximité des installations communautaires
H) Proximité des transports en commun
I) Qualité du voisinage

OUI 8

NON8

\begin{tabular}{|c|c|}
\hline 16 & 84 \\
\hline 16 & 84 \\
\hline 66 & 33 \\
\hline 66 & 33 \\
\hline 16 & 84 \\
\hline 16 & 84 \\
\hline 33 & 66 \\
\hline$\underline{0}$ & 100 \\
\hline 50 & 50 \\
\hline
\end{tabular}


10. Serait-il prêt à débourser davantage pour un terrain?

$$
\text { OUI \& NON\& }
$$
A) Près des centres commerciaux
B) Près de I'hopital et de l'université
C) Plus grand que 70 pi X 100 pi
D) Boisé

$\underline{16}-\underline{50}$

16
-100
$-\underline{0}$

16

84

11. Est-il favorable à une réglementation lors de la construction?

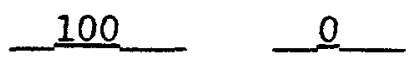

12. Est-il favorable à une réglementation très précise?

$\underline{50}+\underline{50}$

13. Est-il favorable pour réglémenter?

A) les couleurs des revêtements extérieurs

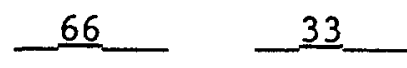

B) Les matériaux de recouvrement extérieurs

84

16

C) I'architecture des maisons

66

33

D) le temps pour effectuer l'aménagement paysager

66

33

Nombre de répondants: 6 


\section{ANNEXX V}

PROFIL DU RESIDENT: $\quad$ Rue des Maristes

1. Age: 38 ans 758 entre 30 et 40 ans

2. Famille: 2 enfants

3. Revenu principal: $\quad \frac{37000}{50 \text { a } 60000} \$$
Revenu familial:

4. Emploi: a) à son compte

b) salarié d'entreprise

c) salarié gouvernemental

d) salarié para-gouvernemental

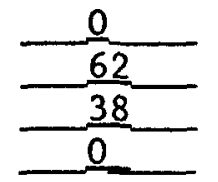

5. Prix prêt à payer pour un terrain: $25000 \quad \$$

6. Superficie de terrain souhaitée: $7000 \mathrm{pi} 2$

7. Prix prêt à payer pour la maison et le terrain:

112500 $\$$

8. Première maison qu'il possède: 258 Deuxième maison qu'il possède: 378 Troisième maison qu'il possède: $\frac{128}{258}$ Quatre et plus $\underline{258}$

9. Points importants pour le choix du quartier:

A) Prix du terrain

B) Prix des maisons

C) Aspect général du quartier

D) Proximité du lieu de travail

E) Proximité des écoles

F) Proximité des centres commerciaux

G) Proximité des installations communautaires

H) Proximité des transports en commun

I) Qualité du voisinage
OUI \&

$\underline{37}$

$\underline{37}$

$\underline{75}$

$-63$

37

$\underline{63}$

25

12

75
NON8

63

63

25

37

63

37

노

87 
10. Serait-il prêt à débourser davantage pour un terrain?.

OUI \& NON\&
A) Près des centres commerciaux
B) Près de 1'hopital et de 1'université
C) Plus grand que $70 \mathrm{pi} \times 100 \mathrm{pi}$
D) Boisé

$\underline{87} \quad \underline{13}$

$\underline{25}-\underline{75}$

$\underline{63}-\underline{37}$

100

11. Est-il favorable à une réglementation lors de la construction?

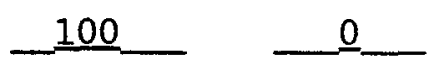

12. Est-il favorable à une réglementation très précise?

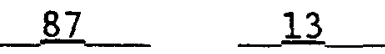

13. Est-il favorable pour réglémenter?

A) les couleurs des revêtements extérieurs

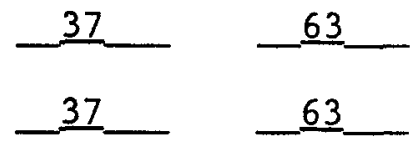

C) I'architecture des maisons

10

$\underline{0}$

D) le temps pour effectuer 1'aménagement paysager

87

13

Nombre de répondants: 8 


\section{ANNEXE VI}

PROFIL DU RESIDENT: Quartier Gobeil (Rivière du Moulin)

1. Age: 35 ans 768 ont entre 25 et 35 ans

2. Famille: 1 à 2 enfants en bas áge

3. Revenu principal:

Revenu familial:

\begin{tabular}{rr}
37 & 000 \\
\hline 50 & 000
\end{tabular}

4. Emploi: a) à son compte

b) salarié d'entreprise

c) salarié gouvernemental

d) salarié para-gouvernemental

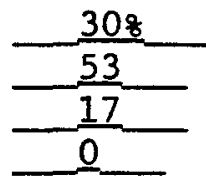

5. Prix prêt à payer pour un terrain: $15,000 \$ 17$ prêt à payer $20000 \$$

6. Superficie de terrain souhaitée: $7000 \mathrm{pi} 2$

7. Prix prêt à payer pour la maison et le terrain: $\quad 80 \quad 000$

$17 \%$ prêt à payer 100 000\$

8. Première maison qu'il possède: $\underline{358}$

Deuxième maison qu'il possède: $\underline{538}$

Troisième maison qu'il possède: 128

9. Points importants pour le choix du quartier:
A) Prix du terrain
B) Prix des maisons
C) Aspect général du quartier
D) Proximité du lieu de travail
E) Proximité des écoles
F) Proximité des centres commerciaux
G) Proximité des installations communautaires
H) Proximité des transports en commun
I) Qualité du voisinage

OUI \& NON\&

59
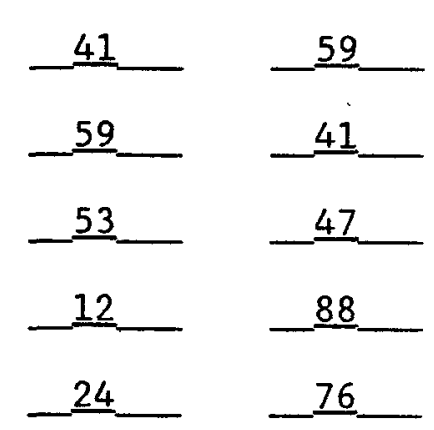

18

82

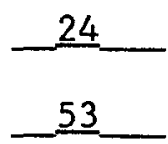

76 
10. Serait-il prêt à débourser davantage pour un terrain?

OUI \& NON\&
A) Près des centres commerciaux
B) Près de l'hopital et de I'université
C) Plus grand que $70 \mathrm{pi} \mathrm{X} 100 \mathrm{pi}$
D) Boisé
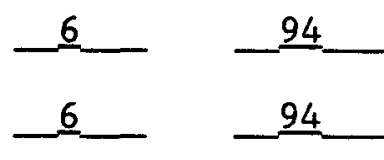

$\underline{70}$

25

$\underline{59}+\underline{41}$

11. Est-il favorable à une réglementation lors de la construction?

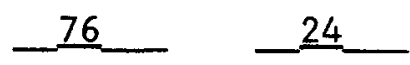

12. Est-il favorable à une réglementation très précise?

$\underline{41}-\underline{48}$

13. Est-il favorable pour réglémenter?
A) les couleurs des revêtements extérieurs
B) les matériaux de recouvrement extérieurs
C) 1'architecture des maisons
D) le temps pour effectuer I'aménagement paysager

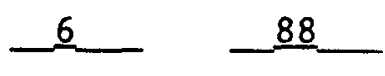

$\underline{6}$

$\underline{30}$

94

50

64

$\underline{50}$

$\underline{50}$

Nombre de répondants: 17 


\section{ANREXE VII}

\section{REGLEMENTS DE CONSTRUCTION POUR}

"LA SEIGNEURIE DES LILAS".

\section{Style d'architecture.}

Les maisons doivent être d'architecture d'aujourd'hui, contemporaine, moderne.

Une maison ne peut être identique à une autre sur une même rue. C'est pourquoi le plan extérieur devra être présenté aux. promoteurs.

2. Revêtements extérieurs de la maison.

a) Matériaux.

Les matériaux de revêtements permis sont la brique d'argile et de béton (brique d'un ton), le stuc ("calcikote"), la pierre de taille.

Les combinaisons de revêtements sont limitées à deux matériaux. Les bardeaux d'asphalte de la toiture devront être neutres et s'harmoniser à l'habitat.

b) Couleur.

Les matériaux de couleurs vives sont proscrits (rouge vif, gros bleu, vert forêt, etc.). 
3. Implantation.

a) Marges latérales.

Les normes de la cité s'appliquent sauf qu'un abri d'auto doit être distant d'au moins 7 pieds de la ligne latérale du terrain.

b) Façade.

La façade de la propriété devra être à vingt-cinq pieds de la ligne avant.

c) Hauteur du solage.

Le dessus du solage devra être de 3 pieds plus haut que le centre de la rue, lorsque le terrain est du même niveau que la rue.

d) Entrée d'automobile.

Pour les terrains face à l'est et à l'ouest, l'entrée de I'auto doit se situer du côté nord.

Pour les terrains face au nord et au sud, l'entrée de l'auto doit se situer du côté ouest.

e) La clôture doit se limiter à la cour arrière et latérale jusqu'au mur arrière du bâtiment.

Les haies sont recommandées au lieu des clôtures. Ce n'est pas une exigence.

4. Maison unifamiliale détachée.

La propriété doit être d'un seul logement.

5. Pente de toit.

Le toit de chaque habitation aura une pente minimale de $3 / 12$, sauf pour le bungalow, où elle devra être d'au moins $5 / 12$. 
6. Aménagement paysager.

Le propriétaire de la maison devra aménager le terrain au plus tard 18 (dix-huit) mois après le début de la construction.

L'aménagement comprendra la pelouse (semence ou tourbe), la plantation de deux (2) arbres devant la propriété, et la surface de l'entrée automobile et piétonnière.

Un lilas (Syringa vulgaris) est exigé devant chaque propriété.

7. Séchoir à linge.

Les cordes à linge sont interdites. Seuls les séchoirs autoportants de 4 mètres carrés maximum sont permis.

8. Limite de temps pour construire.

L'acheteur s'engage à construire dans un délai ne dépassant pas 24 (vingt-quatre) mois. Dans le cas de non-respect de cette clause, le vendeur aura droit de reprise de possession du terrain à condition de rembourser le prix d'achat.

\section{Recommandations (ceci n'est pas une exigence).}

Nous encourageons fortement les propriétaires a construire un garage intégré à la maison pour diverses raisons en plus de son utilité:

1- Meilleur aspect visuel dans le quartier, vu que les automobiles disparaissent du décor.

2- Meilleur aspect visuel, vu la disparition du cabanon pour ranger bicyclette, tondeuse, etc.

3- Meilleur aspect pour la maison car elle parait plus importante.

4- Le coût d'un garage intégré est de 5000 (cinq mille dollars) d'après les contracteurs et une remise est de 1 $500 \$$ (mille cinq cents dollars). Nous pensons que lors de la revente, ce coût sera facilement récupéré. 
bo/Uy/su

CHICOUTIMI METROPOLITAIN IATIONS ET USAGES PREVUS

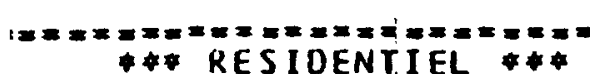

* \# RESIDENTIEL ***

UCTIONS UN IFAMILIALES

UCIIONS BIFAMILIALES

UCTIONS MU

SOUS-TOTAL
SS
ABRIS D.AUTOS
OLOTURE, MUR, HAIE
ENS. MIXTE RES. C COMMUNAUTAIRE ITIONS, AGRANDISSEMENTS

GEMENT, OIVERS, CHANGEMENT D'USAGE LLE INOUVELLES CONSTRUCTIONSI

TIONS, AGRANDISSEMENTS

GEMENT, DI VERS, CHANGEMENT D.USAGE ;OLE

** COMMERCIAL ***

LES CONSTRUCTIONS

TTES CONS, AGRANDISSEMENTS
IGEMENT, DIVERS, CHANGEMENT DOUSAGE

INST ITUTIONNEL COMMUNAUTAIRE

LES CONSTRUCTIONS

ITIONS, AGRANDISSEMENTS
IGEMENT, DIVERS, CHANGEMENT DOUSAGE

*** INOUSTRIEL ***

LES CONSTRUCTIONS

TIESNON AGRANOISSEMENTS

IGEMENT, DI VERS. CHANGEMENT DOUSAGE

IES (TRANSPORT, RECREATIF, SERVICES) *

LES CONSTRUCI IONS

IGEMENT;, DI VERS, CHANGEMENT D*USAGE

DEMOLITIONS
AL OU MOIS: SEPTEMBRE 1988
AL OU MOIS: SEPTEMBRE 1987
BE POUR B6-87-88 SEPTEMBRE
SEKVILE U.UKBANISME - PEKMIS $E$ INSPELIIUNS COMPILATION DU MOIS SEPTEMBRE 1988

COMPILATION CUMULATIVE dePUIS J. NUMBRE UE VALEUR DECLAREE NOUVEAUX
PERMIS NOMBRE DE VALEUR DECLAREE NOU PERMIS

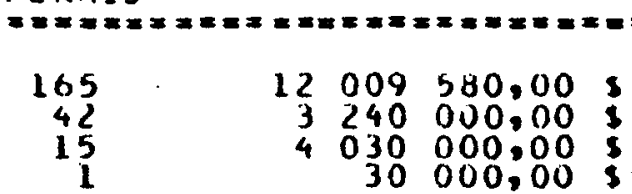

201500,00
232000,00
$510000 \% 00$
0,00

1943500,005 0,008

1000,00

16200,00

0,00

0,00

414195,00

38550,00

0,00

$800,0,005$

$\begin{array}{rr}70 & 000,00 \\ 6 & 000,00\end{array}$

0,008

0,00 s

16
6
9
0

-31
0
0
0
0
0
0
0
1
0
0
0
0
0

2

0
0
0

2
3
0

515000,00
37500,00
$\quad$

$234,000,00$
$1.160,000,00$

$160,000,00$
5000,00

0,00

$\frac{-14894500}{4.5}-\frac{1}{33}$

$\frac{5170409,00}{3980251,003}-\frac{44}{34}$
19309580,005 $0: 00$

$83 \quad 385: 00$

181100,00 0,00

0,00

0,00

$3398990 \% 00$ 451900,00 50000,00

18500,00 0,00 s

1732000,00

1222050,00

39

$755 \quad 740,00$
0,00

612
525
5000,00

14100,00

$801000,00:$

832700,00
197500,00

$-\frac{14}{997}-\frac{12}{35814073,000,005}-$

$37631037,00=$

Defigue: OS - w w w

nom. $34926153,00 \%$ CHEF INSPECTEUR EN 